\title{
Competence in thoracic ultrasound
}

\author{
Tudor P. TOMA 1 *, Marco TRIGIANI ${ }^{2}$, Alessandro ZANFORLIN ${ }^{3}$, \\ Riccardo INCHINGOLO 4, Mauro ZANOBETTI 5, Lucia SAMMICHELI 6, Emanuele G. CONTE 7, \\ Giuseppe BUGGIO ${ }^{8}$, Liliana VILLARI ${ }^{\text {, }}$, Lorenzo CORBETTA ${ }^{10}$, Giampietro MARCHETTI 11
}

\begin{abstract}
${ }^{1}$ University Hospital Lewisham, Greenwich NHS Trust, London, UK; 2 Division of Interventional Pulmonology, Department of Cardiothoracic and Vascular Disease, Careggi University Hospital, Florence, Italy; ${ }^{3}$ Department of Internal Medicine, Bolzano Central Hospital, Bolzano, Italy; ${ }^{4}$ Department of Respiratory Medicine, A. Gemelli University Hospital, Sacred Heart Catholic University, Rome, Italy; ${ }^{5}$ Department of Emergency Medicine, Careggi University Hospital, Florence, Italy; ${ }^{6}$ Sub-intensive Unit, Department of Emergency Medicine, Careggi University Hospital, Florence, Italy; ${ }^{7}$ Department of Respiratory Medicine, C. e G. Mazzoni Hospital, Ascoli Piceno, Italy; ${ }^{8}$ Service of Pneumology, San Bassiano Hospital, Bassano del Grappa, Vicenza, Italy; ${ }^{9}$ Division of Pneumology, AUSL Toscana Nord-Ovest, Apuane Hospital, Massa, Italy; ${ }^{10}$ Unit of Interventional Pulmonology, Department of Experimental and Clinical Medicine, Careggi University Hospital, University of Florence, Florence, Italy; ${ }^{11}$ Division of Pneumology, Spedali Civili, Brescia, Italy
\end{abstract}

*Corresponding author: Tudor P. Toma, University Hospital Lewisham, Greenwich NHS Trust, London, UK. E-mail: t.toma@nhs.net

\section{A B S T R A C T}

Ultrasound examination is traditionally considered a safe and repeatable exam, but its use is highly operator-dependent. Because of this, lack of sufficient operator skills could lead to diagnostic errors and damage to patient safety related to unnecessary tests or interventional procedures. The indications for lung ultrasound include: diagnosis, quantification, and follow-up of different conditions for which acute respiratory failure or chest pain are the main clinical presentation. Clinicians should have theoretical and practical knowledge on: physics and technology of ultrasound, indications and methodology of ultrasound examination, normal thoracic anatomy identification by echography, and detection of signs of pleuro-pulmonary pathology. Consequently, according to international recommendations, core basic skills and minimum training recommendations for the practice of medical ultrasound and image acquisition are needed to ensure competence of clinicians using ultrasound.

(Cite this article as: Toma TP, Trigiani M, Zanforlin A, Inchingolo R, Zanobetti M, Sammicheli L, et al. Competence in thoracic ultrasound. Panminerva Med 2019;61:344-66. DOI: 10.23736/S0031-0808.18.03577-2)

KEY WORDS: Pulmonary medicine - Ultrasonography - Diagnostic imaging - Thorax - Lung diseases - Competence.

$\mathrm{T}$ horacic ultrasound (TUS) applications are increasing and TUS is now used not only by chest physicians, but also by internal medicine, internal therapy unit (ITU) doctors, general practitioners, and various other health care professionals, such as paramedics and physiotherapists. There is almost no direct risk from touching a patient with an ultrasound probe, and this may encourage health care professionals to start using TUS even in the absence of a structured, formal training. However, there is important knowledge related to machine settings, image acquisition and image interpretation, which, if ignored, can lead to serious clinical errors. Thus, there is a need for a simple, but a standard approach to TUS education and competency for health care professionals that are not radiologists. This approach should also be adapted to the local needs and should be evidence based; it should empower doctors to perform effective and safe TUS in a variety of clinical scenarios.

\section{Training guidelines available}

Numerous societies and organizations with responsibilities for medical education have produced guidelines for TUS training. ${ }^{1}$ Perhaps most widely accepted guideline at the European level is the document produced by the European Federation of Societies for Ultrasound in Medicine and Biology (EFSUMB). ${ }^{2}$ This document, with a focus 
on pleural pathology, describes a curriculum and an assessment form, with three levels of competences. Other Societies, such as American College of Chest Physicians, published statement papers describing technical and cognitive elements required for competence in lung ultrasonography in critical care ${ }^{3}$ while intensive care and cardiology Societies have included elements of TUS training in their published curricula..$^{4,5} \mathrm{~A}$ very comprehensive system for TUS training and accreditation has been described by the Australian doctors. ${ }^{6}$ European Respiratory Society has published a monography on thoracic ultrasound, ${ }^{7}$ but there are no formal guidelines and standards yet from the ERS. Italy is largely following the evidence-based recommendations for point of care chest ultrasound produced by the International Liaison Committee on Lung Ultrasound (ILC-LUS). ${ }^{8}$

Nevertheless, most of these guidelines are based on expert opinion. This is mainly because there are still not yet available a clear set of evidence-based studies to answer specific questions regarding the best ways to train non-radiologists to do TUS. A recent paper screened 7796 studies on ultrasound education and ended up including only 16 studies in a meta-analysis, ${ }^{9}$ of which only one was a randomized controlled trial. The analysis concluded that so far "it was not possible to construct clear guidelines for the future education and certification in clinical lung ultrasound, but the use of different hands-on training facilities tends to contribute to different aspects of the learning process."

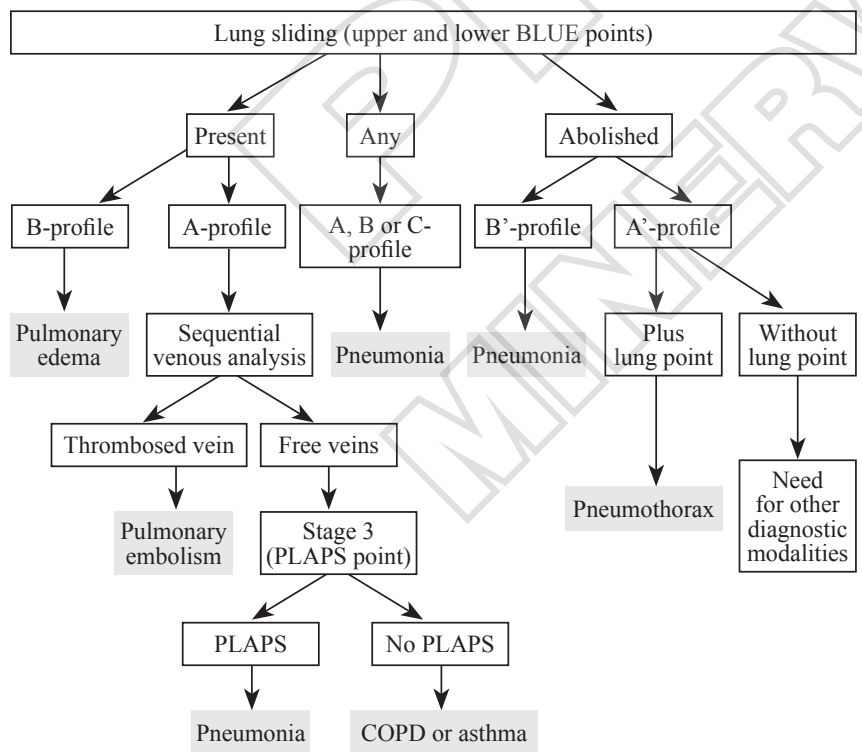

Figure 1.-The BLUE protocol decision tree (modified from Lichtenstein and Mezière). ${ }^{12}$

\section{Knowledge of the diseases and image interpretation in the clinical context}

Thorax and lung ultrasound (LUS) has gained importance in clinical activity, which is especially true in the setting of point of care ultrasound (POCUS). ${ }^{8}$ They represent an integration to the physical examination, to recognize specific ultrasonic signs that narrow the list of potential diagnoses in clinical settings. ${ }^{10}$ Some scanning protocols as the BLUE, FALLS, and C.A.U.S.E. have been used in patient management as algorithms using ultrasound sign combinations, to achieve accurate diagnoses ${ }^{11,12}$ (Figure 1,2,3).

Knowing the medical history of the patient, the current symptoms, the signs form physical examination and imaging findings are crucial in obtaining a correct interpretation of ultrasound findings of the thorax and lung. ${ }^{13}$

\section{Indications}

The indications for lung ultrasound include: diagnosis, quantification, and follow-up of different conditions for which acute respiratory failure or chest pain are the main clinical presentation. ${ }^{4}$

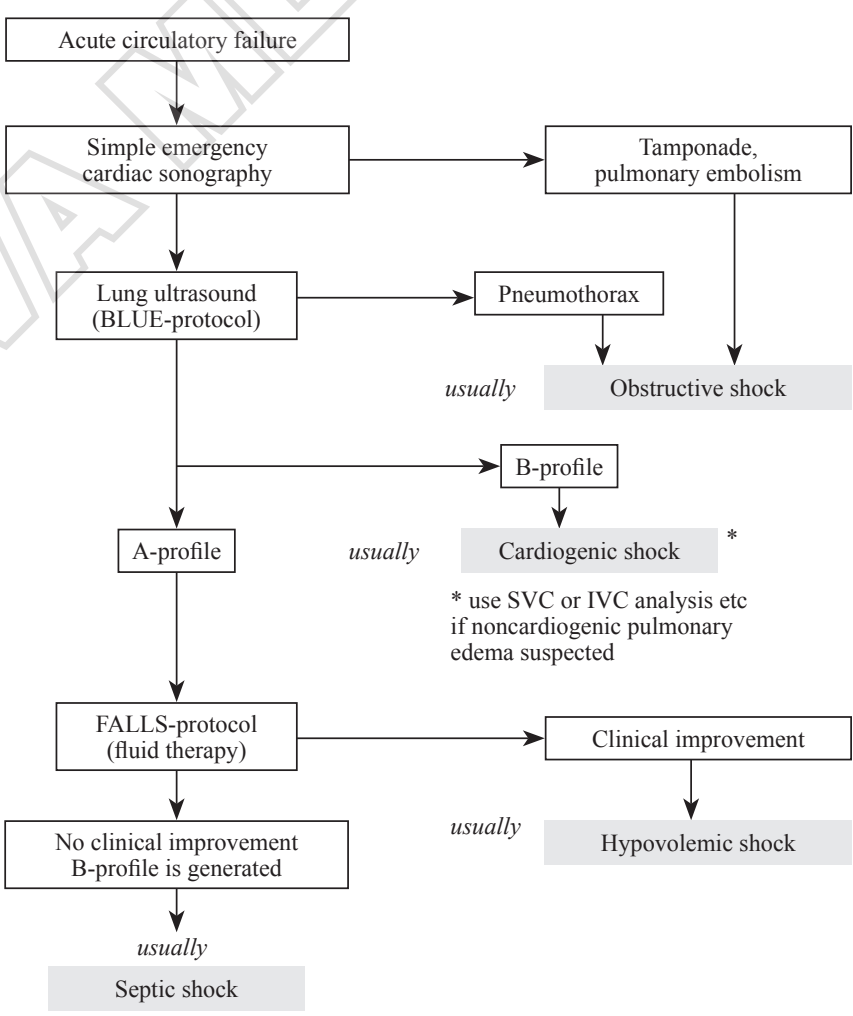

Figure 2.-The FALLS protocol decision tree (modified from Lichtenstein). ${ }^{11}$ 


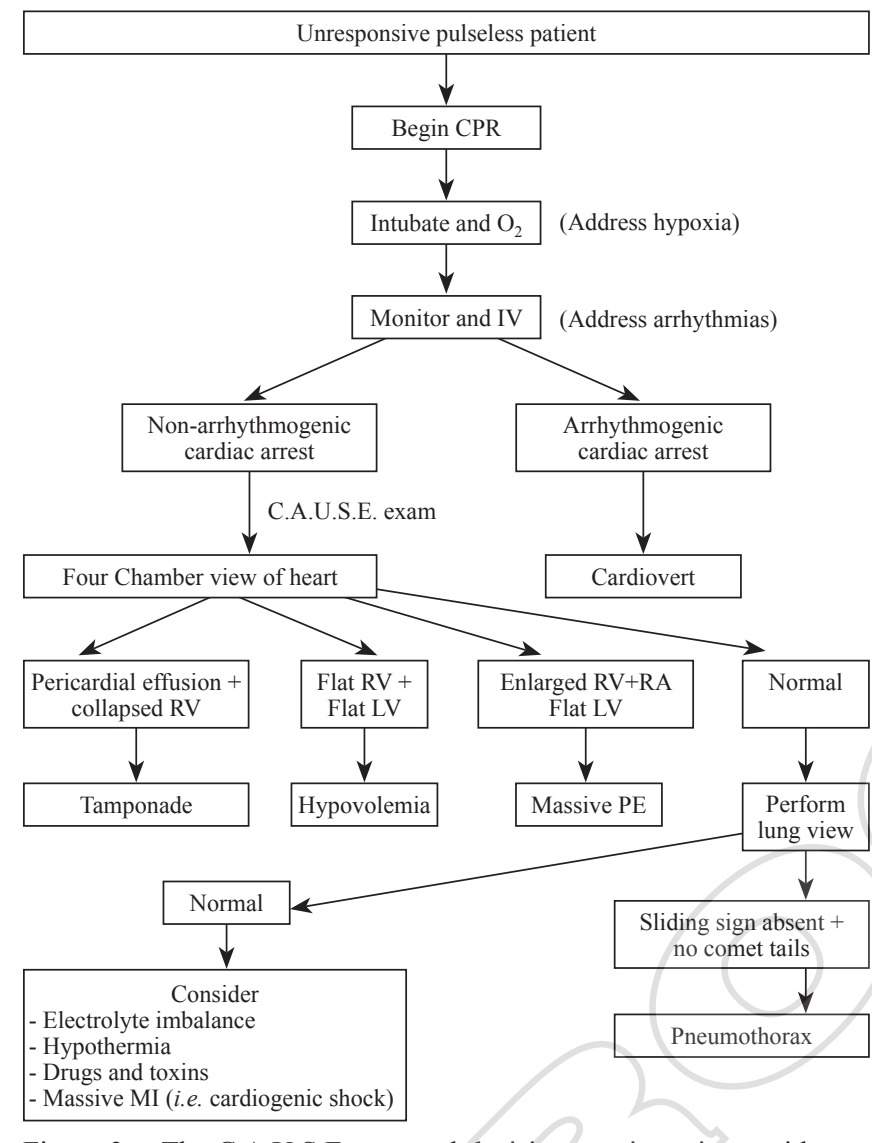

Figure 3.-The C.A.U.S.E. protocol decision tree in patients with cardiac arrest.

The three main fields of application for lung ultrasound are:

- interstitial syndromes;

- alveolar syndromes;

- pleural syndromes,

and appropriate use for procedures such as:

- guidance for diagnostic and therapeutic thoracentesis;

- placement of thoracostomy tubes.

\section{Advantages}

For critically-ill patients, bedside thoracic ultrasound is faster, does not require transfer of patients, and is less resource-demanding than CT scanning. ${ }^{15}$

It has also several advantages over traditional radiographic imaging of the pleura including: absence of radiation, better portability, real-time imaging, and the ability to perform dynamic imaging.

US is substantially better than physical examination for determining the location of pleural fluid, for differ- entiating pleural fluid from lung consolidation, for the diagnosis of pleural thickening and pleural masses and is associated with a lower rate of complications during thoracentesis.

\section{Disadvantages}

TUS is an operator dependent technology. Focused and supervised training is needed to ensure that the operator correctly interprets the sonographic findings Inadequate training may increase the risk of complications. TUS does not rule out any pulmonary abnormalities that do not reach the pleura.

Ultrasound is not as good as CT imaging for evaluation of the underlying lung parenchyma in the setting of complex pleural and lung parenchymal disease and ultrasound guidance is not as good as guidance by CT imaging for complicated interventional procedures, such as empyema drainage with a pigtail catheter or biopsy of pleural masses. ${ }^{13}$

\section{Semeiotics ultrasound}

As in the myth of Plato's cave, in which reality is perceived through the projection of shadows, in thoracic ultrasound lung's anatomy can be assessed through the interpretation of indirect signs or artifact patterns. Indeed, the normal pleural line and A, B, C, E, and Z lines (also known as false B lines) are artifacts with specific characteristics. Lung consolidation and a pneumothorax have established sonographic patterns. Evaluation of real anatomic images is possible in pleural effusion (PE) with total lung atelectasis or in complete lung consolidation, with a direct visualization of the diaphragm. ${ }^{13}$

The normal pleural line is a thin, bright, hyperechoic horizontal linear structure, visualized between the rib shadows. The sonographic signs generated by or below the pleural line are the following.

\section{Lung sliding}

Also known as lung gliding, or the lung sliding sign, it is a sign created by the movement of the parietal and visceral pleura during respiration. Lung sliding during M-mode examination will be characterized by a pattern referred to as "the seashore sign": a wave-like pattern located above (towards the chest wall) and a granular pattern below (Figure 4). Lung sliding is an indirect sign indicating contact of the visceral pleura to the parietal pleura. When air or fluid separates the two pleural layers as in a pneumothorax or pleural effusions, the sliding sign disappears. ${ }^{16}$ 
Figure 4.- Linear probe: sliding (single arrow) and A line (double arrow) are visible.

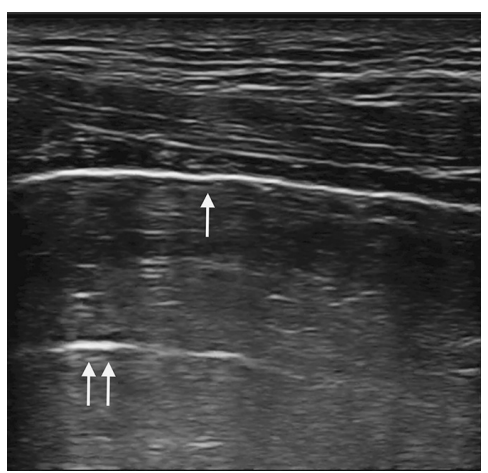

\section{Lung pulse}

The lung pulse is a low amplitude rhythmic sliding caused by cardiac pulse transmitted to the lung and hence to the pleural surface. It is best seen in areas closer to the pericardium and the heart. The lung pulse is caused by transmission of heart impulse through consolidated, motionless lung. The presence of air between visceral and parietal pleura prevents transmission of either horizontal or vertical heart impulses to the parietal pleura. Therefore, visualization of a lung pulse excludes a pneumothorax. However, the lung pulse is only of significance if it is actually present; absence of a lung pulse is not diagnostic of a pneumothorax. ${ }^{17}$

\section{Lung point}

It is a pathognomonic sign of a pneumothorax. It is a transition point from an area with absence of lung sliding, Blines, lung pulse and presence of only horizontal reverberation artefacts into an area of lung sliding. ${ }^{18}$ It indicates a zone where the lung comes in contact with the parietal pleura intermittently. It can be found by moving the probe along different intercostal spaces. However, a lung point is not always present, such when a large pneumothorax causes extensive lung collapse and the lung does not reach at all the pleural surface. In M-mode, "stratosphere sign" or "barcode sign" pattern can be seen when pneumothorax is present (Figure 5).

Lines

A lines represent horizontal reverberation artifacts seen below the pleural line characterized by the same spacing between the probe and the pleural line and generated by air below the pleura. ${ }^{19}$ These lines are commonly seen in healthy individuals and may be erased (by B lines) or enhanced (in the presence of pneumothorax) (Figure 6, 7).

$\mathrm{B}$ lines or ultrasound lung comets are defined as dis-

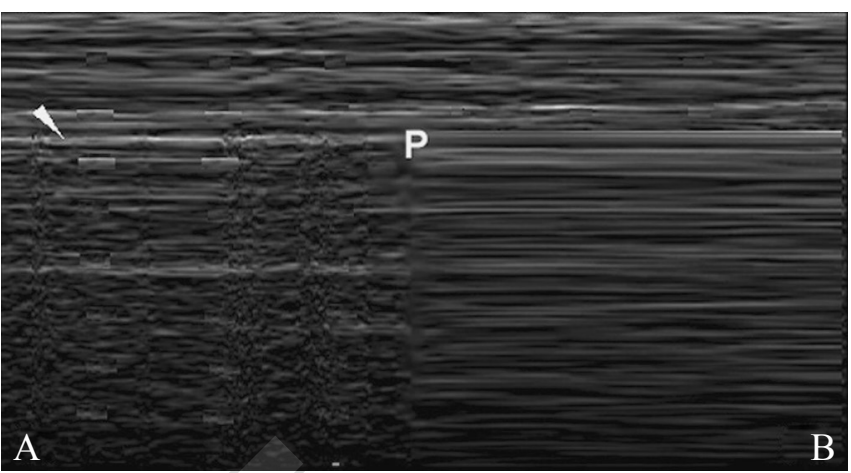

Figure 5.-Linear probe: lung point $(\mathrm{P})$ divides the seashore $(\mathrm{A})$ and barcode sign (B); sliding (single arrow) is well visible in the seashore sign.

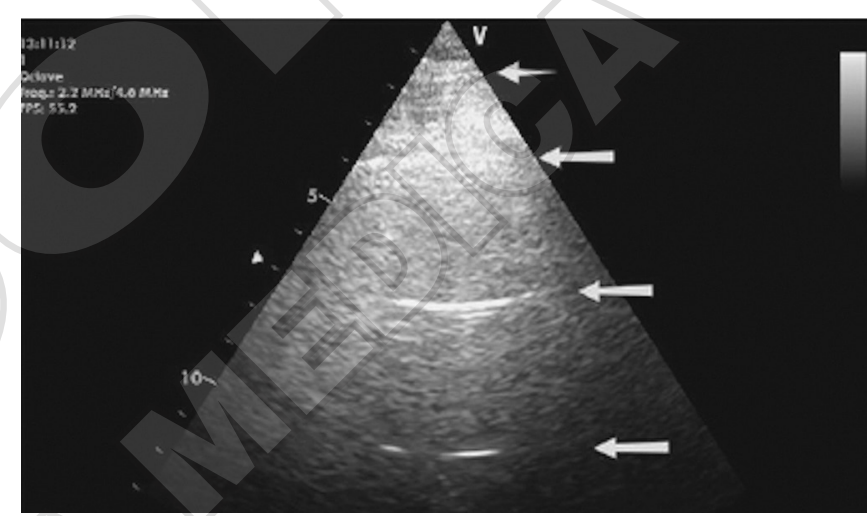

Figure 6.-Convex probe: A lines (arrows) are reflected with the same spacing between the probe and the pleural line (fine arrow).

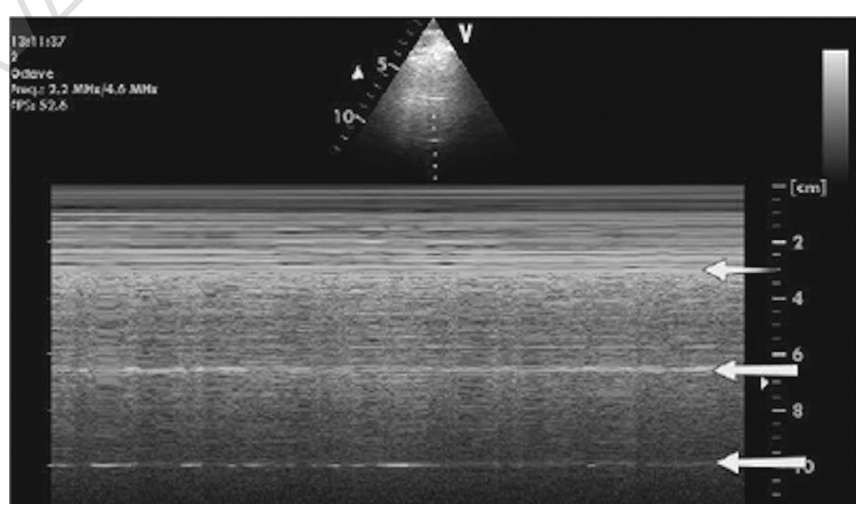

Figure 7.-Convex probe: A lines (arrows) and pleural line (fine arrow) in M-mode vision.

crete laser-like vertical hyperechoic reverberation artefacts that arise from the pleural line (previously described as "comet tails"), which extend to the bottom of the screen without fading and move synchronously with lung slid- 
ing. (Figure 8,9) The artefact consists of a trail of dense echoes that resembles a distally oriented comet-tail. ${ }^{20} \mathrm{~B}$ lines indicate filling of intralobular or interlobular septa and are often seen in pulmonary edema and interstitial lung diseases. ${ }^{8}, 11$ Thickened $\mathrm{B}$ lines may fuse together to form coalescent B lines representing peripheral lung ground glass opacities seen in high resolution computed tomography $(\mathrm{CT}) .8,11,21$

$\mathrm{C}$ lines are defined as hypoechoic subpleural focal images generated by condensed lung tissue, without a visceral pleural line gap. C lines are not "lines" but are named as

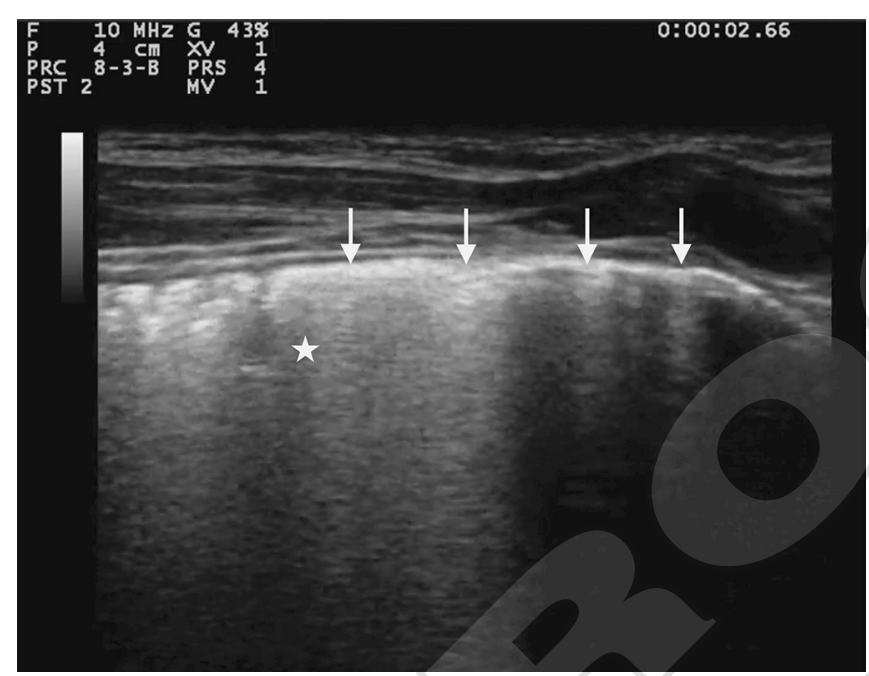

Figure 8.-Linear probe: B lines (arrows) and small lung consolidation (star) without pleural line.

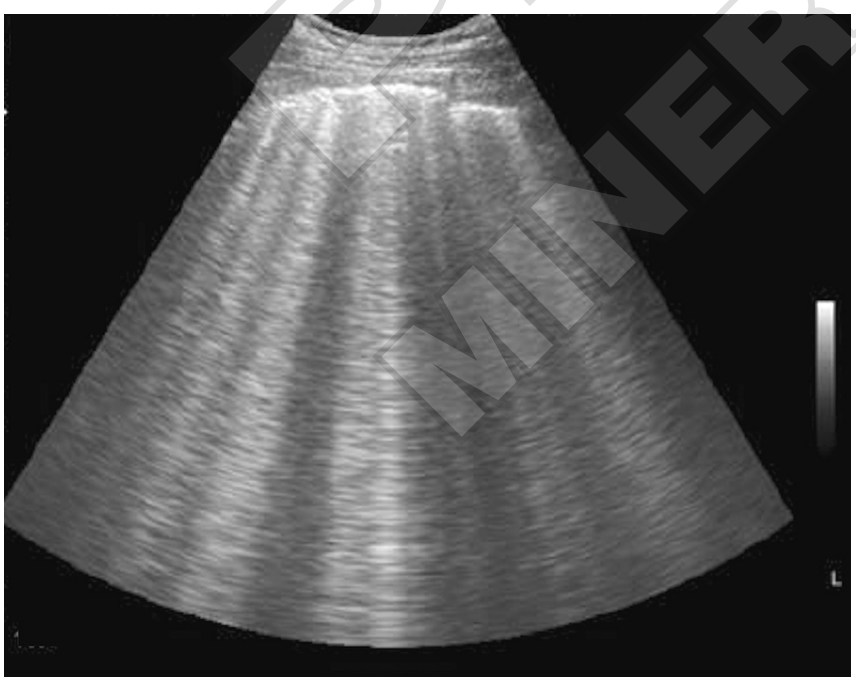

Figure 9.-Convex probe: B lines as laser-like vertical hyperechoic reverberation artefacts. such for the sake of consistency with nomenclature standards (Figure 10).

$\mathrm{E}$ and $\mathrm{Z}$ lines (false $\mathrm{B}$ lines) are vertical artifacts originating from to the pleural line and may be mistaken for true B lines. However, such lines represent different entities. E lines appear with subcutaneous emphysema. E lines are vertical lines seen when there is gas trapped in the subcutaneous space. These lines do not arise from the pleural line, but from the subcutaneous tissue; given the gas does not move, they are not synchronous with respiratory movements. E lines are well-defined and can also erase A lines, so they can be mistaken for true $\mathrm{B}$ lines. ${ }^{20} \mathrm{Z}$ lines are common artifacts seen in more than $80 \%$ of the population and may be mistaken for coalescent $B$ lines described above. $\mathrm{Z}$ lines are vertical, bundle-like shaped lines arising from the pleural line; however, they are ill-defined, they do not erase A lines, and are not perfectly synchronous with respiratory movements. ${ }^{15}$

Lung consolidation is a subpleural echo-poor area of the lung with a tissue-like sonographic appearance. The degree of observed consolidation depends on the extent of air loss and fluid and/or tissue predominance. It is related to pneumonia or atelectasis. Loss of air allows evaluation of the bronchial and the vascular structures inside the lung parenchyma. ${ }^{22}$ When the lung is compressed by a surrounding pleural effusion, it appears hyperechoic, or tissue dense, and frequently exhibits a characteristic flapping movement with the respiratory cycle, or may appear to float in the pleural effusion. (Figure 11, 12).

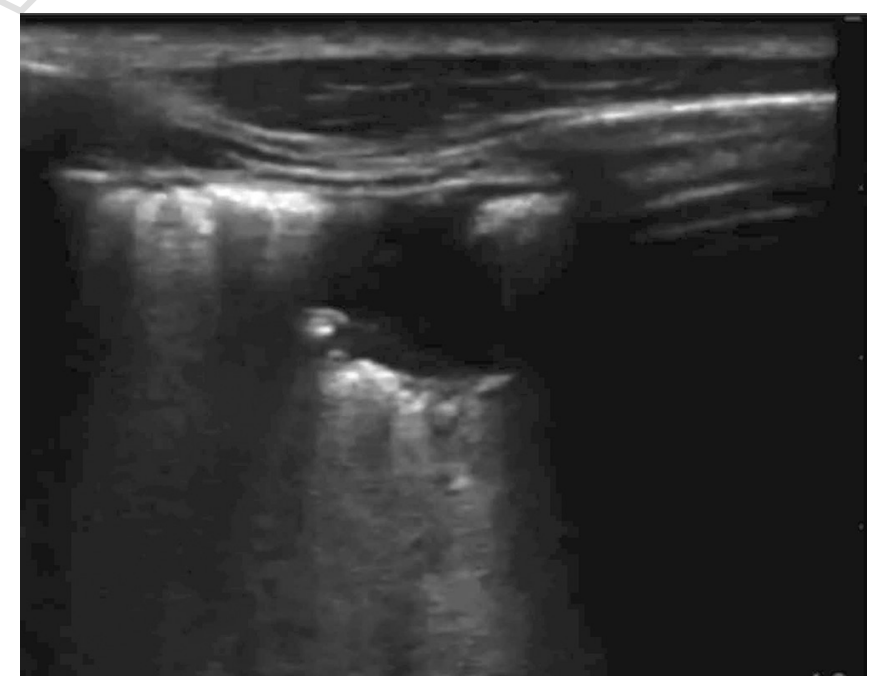

Figure 10.-Linear probe: hypoechoic subpleural focal images without visceral pleural line gap. 


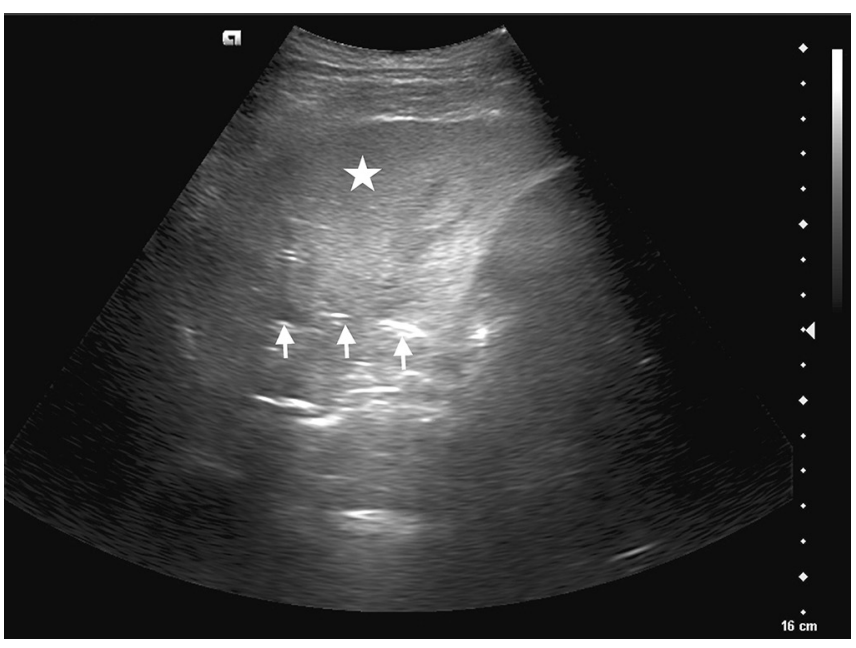

Figure 11.-Convex probe: consolidated hyperechoic lung (star) whit air bronchogram (arrows).

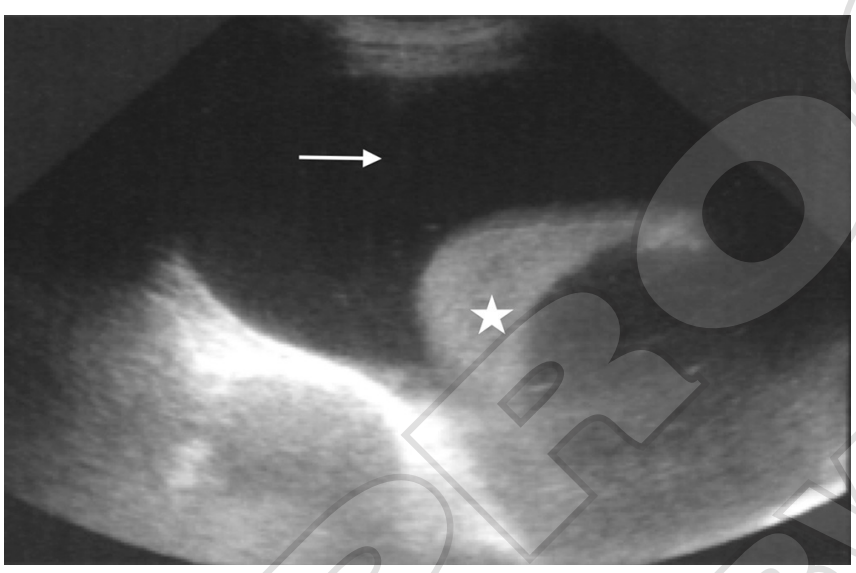

Figure 12.-Convex probe: the hyperechoic lung (star) appears compressed by a surrounding pleural effusion (arrow).

\section{Pleural fluid}

Usually appears as an anechoic (black), or hypoechoic area (compared to the liver) within the thoracic cavity, it is changing its shape with respiratory cycling, and that can include echogenic material (septations, cellular debris, fronds) (Figure 13). A hemothorax and an empyema fluid can be isoechoic with the liver and can have no dynamic changes with respiration. The presence of air and fluid together, due to hemopneumothorax, may present a complex sonographic picture.

\section{Diaphragm and subdiaphragmatic recesses}

The diaphragm typically appears as an echogenic line, approximately $1 \mathrm{~mm}$ thick, with a downward movement in

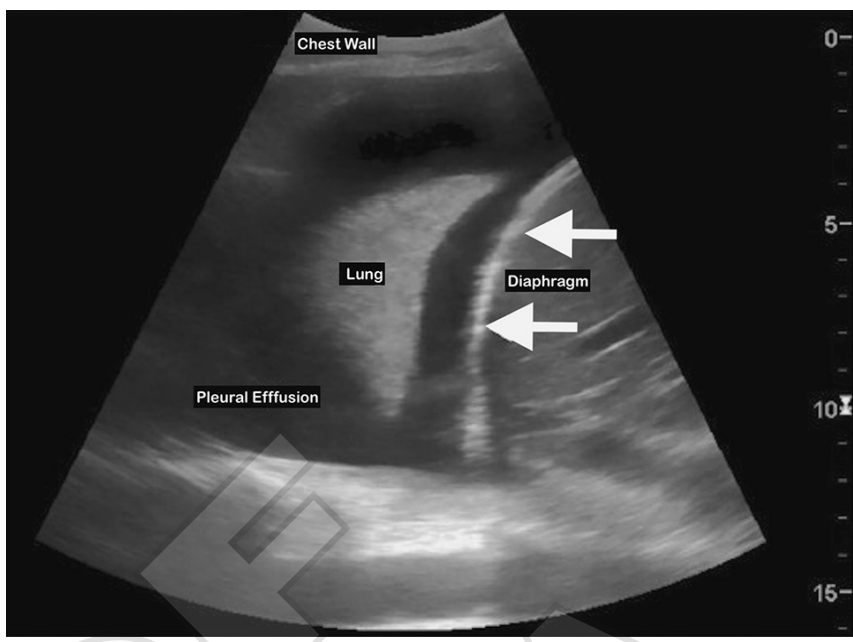

Figure 13.-Convex probe: consolidated lung surrounded by hypoechoic pleural effusions; the diaphragm (arrow) is clearly visible as well as the liver due to effusion.

inspiration (Figure 13). As a general rule, when the patient is sitting, the diaphragm is located caudad to the $9^{\text {th }}$ rib. The splenorenal and hepatorenal recesses are identified by finding the liver or spleen craniad and the kidney caudad to the respective recess. ${ }^{20}$

The morphologic characteristics of a pleural malignancy can include: diaphragmatic and parietal pleural nodules, pleural thickening $>1 \mathrm{~cm}$, or hepatic metastasis; however, ultrasound findings alone are usually not sufficient to make a diagnosis of pleural malignancy. ${ }^{23}$

\section{Interstitial syndromes}

Interstitial syndrome is due to diffuse involvement of the interstitium of the lung and impairment of the alveolocapillary exchange capacity as a consequence of thickened interlobular septa, or presence of extravascular lung water, which can lead to severe respiratory failure.

The sonographic diagnosis is established after the visualization of multiple B lines. A semiquantitative approach for the diagnosis of interstitial syndrome is based on a count of the number of B lines from zero to ten in each rib space, or, if confluent, on an assessment of the percentage of the rib space occupied by $\mathrm{B}$ lines divided by ten. A single $\mathrm{B}$ line in isolation, or a B line pattern confined to the last intercostal space, is unlikely to be indicative of any pathology. Presence of three or more B lines in a longitudinal plane between two ribs is defined as a positive region with an interstitial syndrome.

"White lung" consists of a completely white echographic 


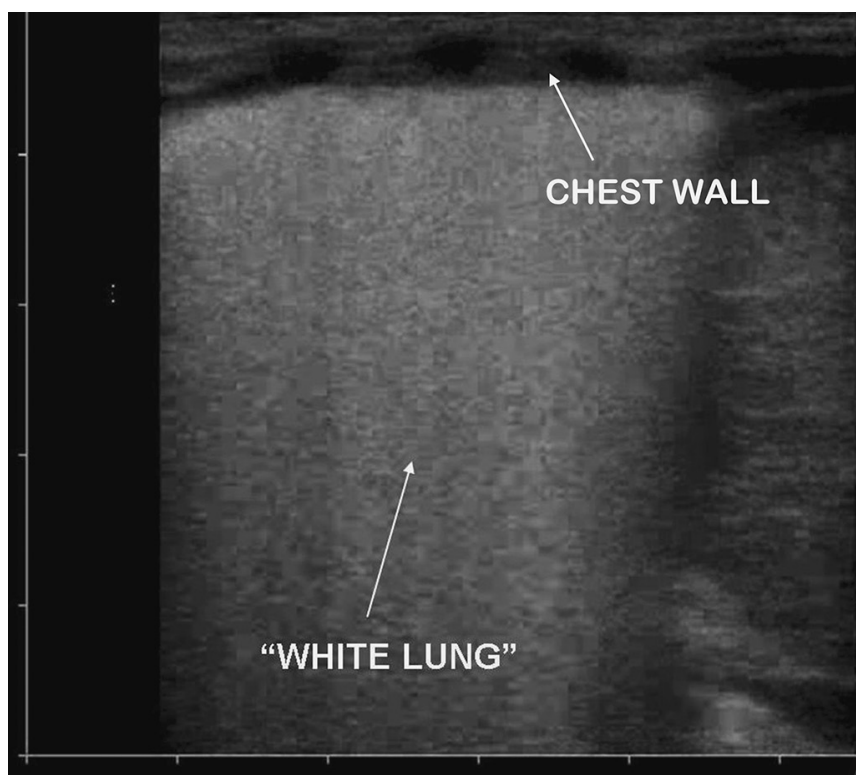

Figure 14.-Linear probe: white echographic lung with coalescent Blines and no horizontal reverberation.

lung, with coalescent B-lines, and no horizontal reverberation (Figure 14). Observation of the B line pattern has an immediate effect on the real-time diagnostic process of critically ill and dyspneic patients. ${ }^{8}$

The term "B-pattern" should be used in the description of multiple B lines in patients with interstitial syndrome. A positive examination with a sonographic diffuse interstitial syndrome allows bedside distinction between a cardiogenic versus a respiratory cause of acute dyspnea.

Multiple diffuse bilateral B lines can indicate an interstitial syndrome due to the following conditions:

- pulmonary edema of various causes;

- interstitial pneumonia or pneumonitis;

- diffuse parenchymal lung disease (pulmonary fibrosis).

In a diffuse parenchymal lung disease (pulmonary fibrosis), in contrast to cardiogenic pulmonary edema, the primary sonographic sign to be identified is the presence of multiple B lines with a nonhomogeneous distribution, and often a pleural line with abnormalities (irregular, fragmented pleural line) plus occasional subpleural abnormalities (small echo-poor areas).

In acute respiratory distress syndrome (ARDS), in contrast to cardiogenic pulmonary edema, the sonographic findings can include the following:

- anterior subpleural consolidations;

- absence or reduction of lung sliding;
- "spared areas" of normal parenchyma;

- pleural line abnormalities (irregular thickened fragmented pleural line);

- nonhomogeneous distribution of B lines.

A focal (localized) sonographic pattern of interstitial syndrome can be seen in the presence of any of the following:

- pneumonia and pneumonitis;

- atelectasis;

- pulmonary contusion;

- pulmonary infarction;

- pleural disease,

- neoplastic disease.

Alveolar syndromes

Alveolar syndromes include alveolar consolidation due to fluid in the alveoli as transudate, exudate, pus, blood, sweet or saline water, or any saline solution, but include also atelectasis (collapsed alveolar units secondary to bronchial obstruction, dynamic airway compression from weakened distal airways or reduced FRC, compression by surrounding effusion). Lung consolidations may therefore have a variety of causes including infection, pulmonary embolism, lung cancer and metastasis, compression atelectasis, obstructive atelectasis, and lung contusion.

The sonographic sign of lung consolidation is a subpleural echo-poor region due to fluid-filled alveoli that give rise to a tissue-like appearance on the ultrasound called "hepatization" because the lung's density and pattern resemble that of the liver. It is named "alveolar" as opposed to the "interstitial" type of syndrome, in which the alveoli still contain air.

Ultrasound is a clinically useful tool to rule in each condition (but does not rule out consolidations that do not reach the pleura) through evaluation of:

- air bronchogram(s);

- fluid bronchogram(s);

- vascular pattern within the consolidation;

- presence of comet-tail reverberation artifacts at the far-field margin;

- quality of the deep margins of the consolidation.

Sonographic air bronchograms, which suggests an infiltrative process with alveolar consolidation, are tubular hyperechoic artifactual structures longitudinal to the beam or punctiform, if transverse within a tissue (Figure 15).

On M-mode, these structures can be further characterized as "dynamic or static."

Static bronchograms produce straight lines, whereas dynamic bronchograms produce a sinusoidal pattern. Static 


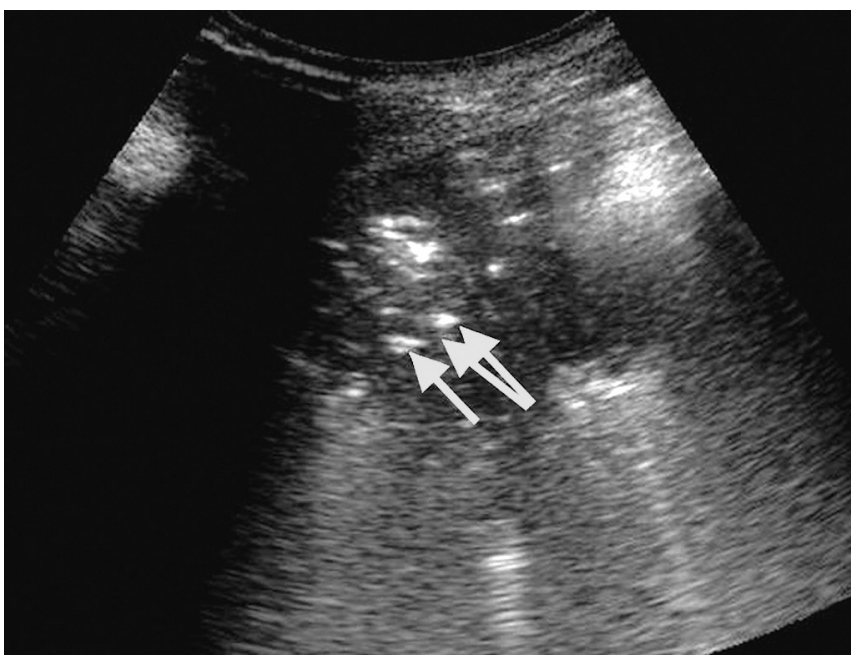

Figure 15.- Convex probe: static air bronchogram (arrows) appear as straight lines, whereas dynamic bronchograms produce a sinusoidal pattern.

air bronchograms were seen in most atelectasis and in one third of patients with pneumonia. Dynamic air bronchograms indicate pneumonia, distinguishing it from atelectasis. 12

Fluid bronchograms are anechoic tubular structures representing fluid-clogged bronchi seen within relatively echogenic collapsed lung. The absence of Doppler flow confirms that these are not vessels, but airways ${ }^{24}$ (Figure 16).

Vascularity is normally present in consolidation. In neoplasia there will be increased vascularity with a disorganized appearance.

In inflammatory pulmonary processes lung sliding is often not present, as consequences of reduced lung expansion and adhesions to the pleura; moreover, the border between consolidated and aerated lung is not sharp (Figure 17).

Abolished lung sliding with a lung pulse and a high and akinetic cupola are immediate signs of acute atelectasia, whereas alveolar consolidation with static air bronchogram and the heart sign (massive visualization of the heart at the anterior wall) are delayed signs.

The repeatability of sonographic examinations in critically ill patients with pneumonia allows monitoring of the effect of therapies by observing the change in the sonographic aspect of the lung, from the alveolar pattern of the consolidated lung to the interstitial pattern, with a decreasing density and number of B-lines, to the final step of normal pattern sign. Interstitial and alveolar patterns can often coexist in the same condition. ${ }^{8}$

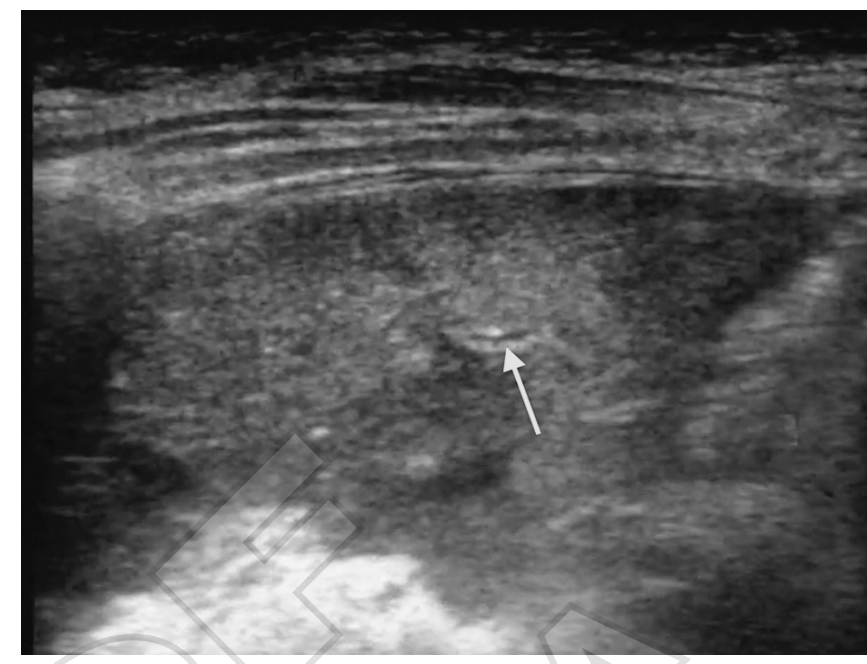

Figure 16.- Linear probe: fluid bronchogram (arrow) appear as anechoic tubular structures representing fluid-clogged bronchi. Absence of Doppler flow confirms the presence of airway.

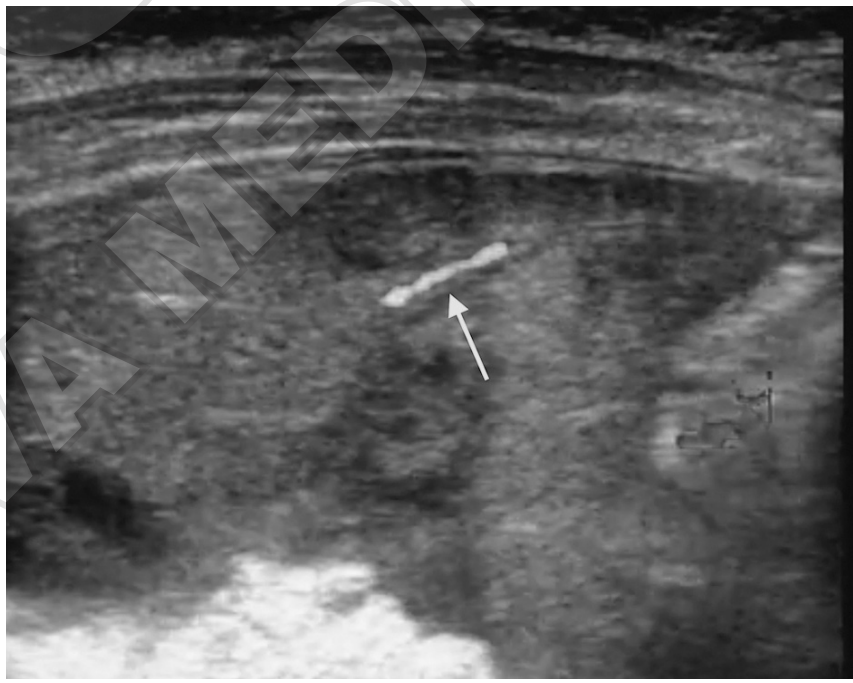

Figure 17.- - Linear probe: normal vascularity (arrow) in consolidated lung.

\section{Pleural syndromes}

\section{Pneumothorax}

The ability to rule out pneumothorax quickly is a critical application of pleural ultrasonography, that has greater specificity and equal sensitivity than chest radiography.

The four key features of a pneumothorax are the following:

- absence of lung sliding and "seashore sign" (Figure $18)$; 


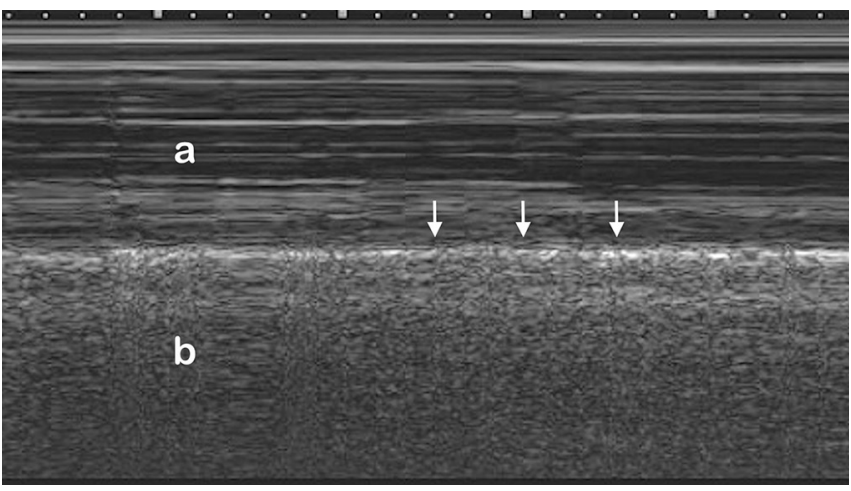

Figure 18. - Convex probe: seashore sign in M-mode vision; pleural line (arrows) divided the chest wall (a) from the lung (b).

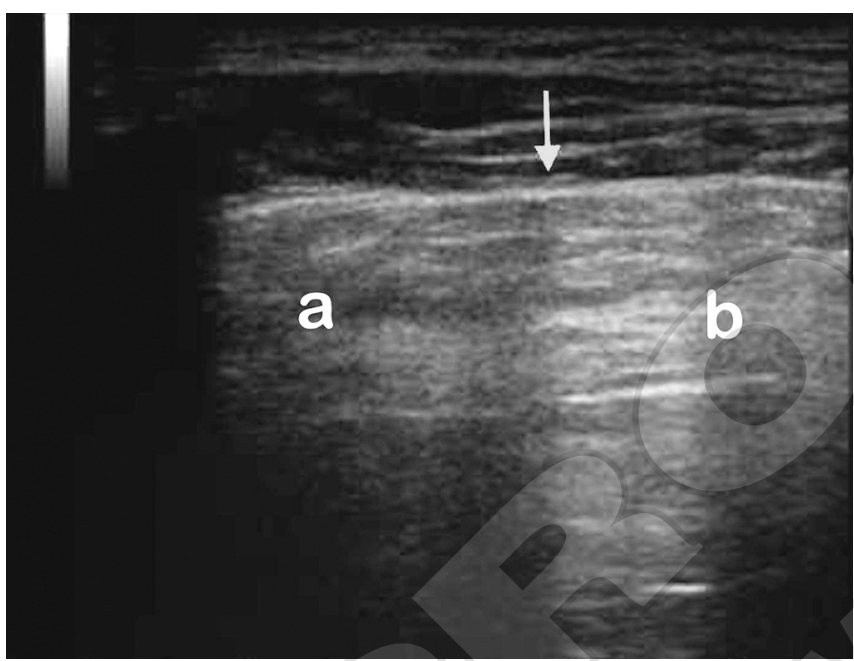

Figure 19.- - Linear probe: lung point (arrow) in M-mode vision divided PNC (a) from lung (b).

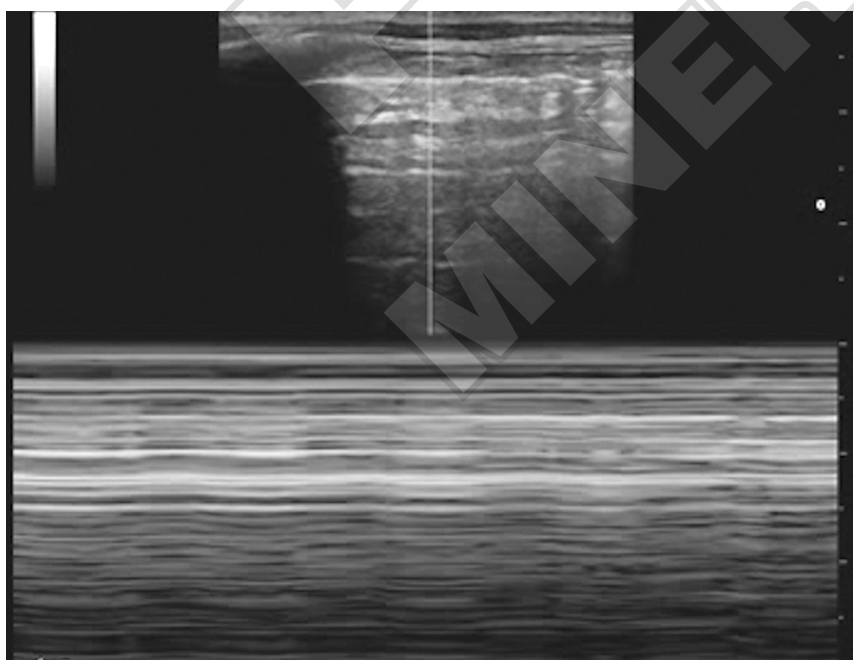

Figure 20.-Linear probe: barcode sign in M-mode vision.
- absence of B lines (Figure 19);

- presence of lung point and "barcode" sign (Figure 19, 20);

- absence of lung pulse.

The presence of lung sliding rules out pneumothorax, but the opposite is not true because it may be absent in other conditions such as atelectasis, pleural thickening, extensive pulmonary fibrosis bullae, contusions, and pleural symphysis that can result in false positives.

In a patient with COPD, the ultrasound appearance of emphysematous areas can mimic a pneumothorax. ${ }^{25}$

In addition, subcutaneous emphysema can lead to sonographic artifacts, such as E lines that are created by the subcutaneous air and mimic B lines.

Further studies are needed to determine if information obtained by assessing the lung tissue with additional sonographic modes such as tissue Doppler may facilitate the diagnosis when a diagnostic doubt exists. ${ }^{26}$

\section{Pleural effusions}

Pleural effusions are the result of increased capillary permeability, decreased colloid osmotic pressure or increased pleural capillary hydrostatic pressure, alterations of lymphatic drainage and passage of peritoneal fluid from diaphragm.

According to the Light criteria, pleural effusion can be divided into transudate or exudate by ratio proteins or LDH between pleural fluid and plasma. ${ }^{27}$ Transudate pleural effusion can be caused by alteration of hydrostatic $o$ colloid pressure, as in the heart failure, hypoalbuminemia or nephrotic syndrome. Exudative pleural effusion can be caused by alteration of capillary permeability as in the inflammation, infections, lung or pleural cancer.

A pleural effusion is a good acoustic window that enhances pleural anomalies or lung lesions and the diaphragm line, so ultrasound is superior to chest radiography in detecting it. In addition, ultrasound can detect the size and the types of a pleural effusion. It is important to study the entire chest to detect pleural effusions that may be loculated and will not collect in dependent areas of the chest. ${ }^{28}$

There are three cardinal rules for diagnosing a pleural effusion on ultrasound (Figure 12):

1. identification of the anatomic boundaries of a pleural effusion:

a. the chest wall;

b. the diaphragm and subdiaphragmatic organs: liver (right side), spleen (left side), and the kidneys;

c. lung should be identified and distinguished from the pleural effusion; 
2. identification of the relatively anechoic space that will constitute the pleural effusion.

3. identification of the dynamic changes:

a. a flapping lung refers to the undulating movement of the compressed lung within the pleural effusion (jellyfish sign $)^{29}$ (Figure 20). The lung may not be compressed, and a flapping lung may be absent with small effusions;

b. the curtain sign refers to aerated lung that moves into the scanning field and partially obscures the pleural effusion and adjacent compressed air during inspiration ${ }^{30}$ (Figure 21);

c. the sinusoid sign reflects the respiratory motion of the visceral pleura on M-mode as it moves toward and away from the chest wall with each breath; it represents movement of the pleural surface within the fluid-filled pleural space. ${ }^{19}$

US can determine the size of a pleural effusion using

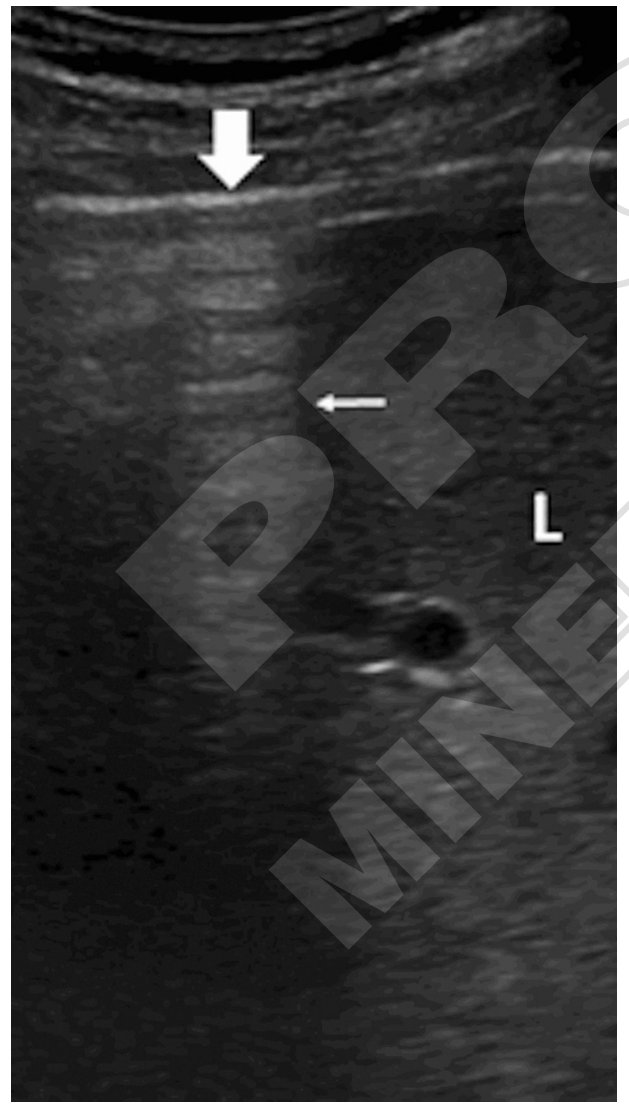

Figure 21.-Convex probe: liver and dip. The curtain sign (small arrow) is the US characteristics of the inferior aspect of the lung field, corresponding to the costophrenic recesses and peripheral lung bases. Liver (L) and diaphragm are partially covered by the lung. Pleural lines are present (big arrow). different protocols (pleural spaces, mathematical formulas) and can classify them as small, moderate, or large. ${ }^{31}$

Pleural effusions have different echogenicity on ultrasound according with underlying causes.

Transudative effusions appear as a space (usually anechoic) between the parietal and visceral pleura that changes according to patient's position (Figure 12). Lung appears with different degrees of compression and, depending on the amount of fluid, it also moves with breathing ("sinusoid sign") or with heart pulsations ("pulse sign").

Exudate effusions show echoes that suggests the presence of debris (cell, fibrin blood) (Figure 22, 23).

Fibrin can be observed in exudative effusions, but the amount, the distribution and the organization in septa or locules is different from patient to patient, depending on the cause of the effusion and the time from onset (Figure

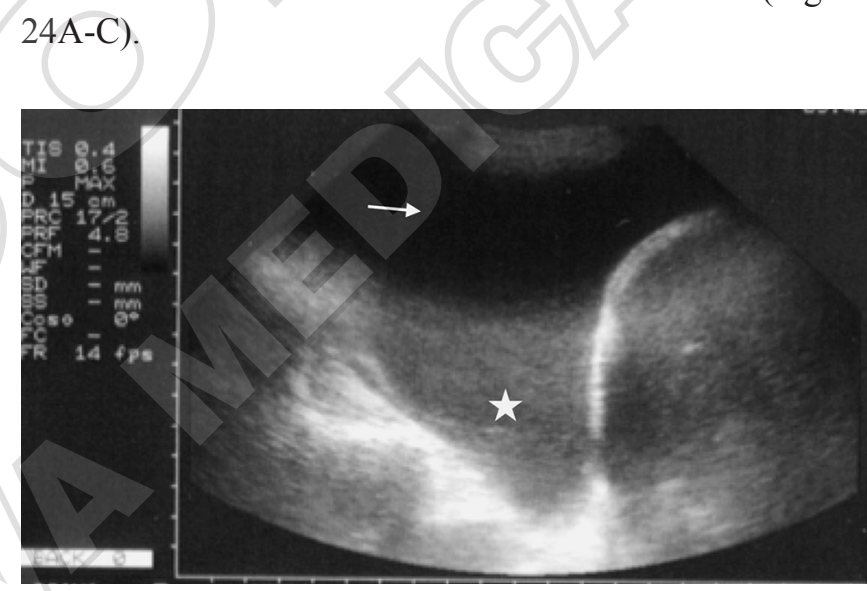

Figure 22.-Convex probe: hematocrit sign; the corpuscular hyperecogenic sediment (star) is visible in the pleural effusions. Sediment change with the body position according to gravity law.

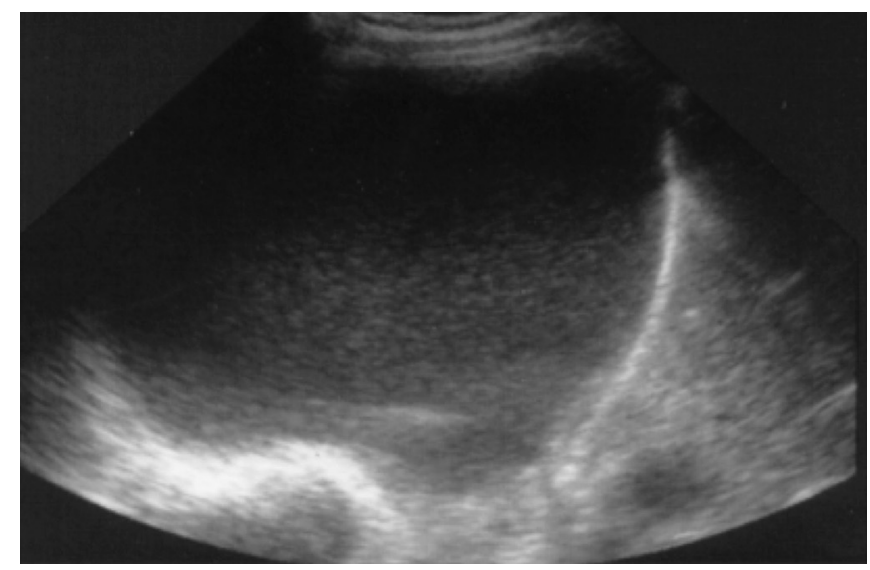

Figure 23.-Convex probe: different and mobile echoes that suggest the presence of debris are seen in the contest of pleural effusion. 

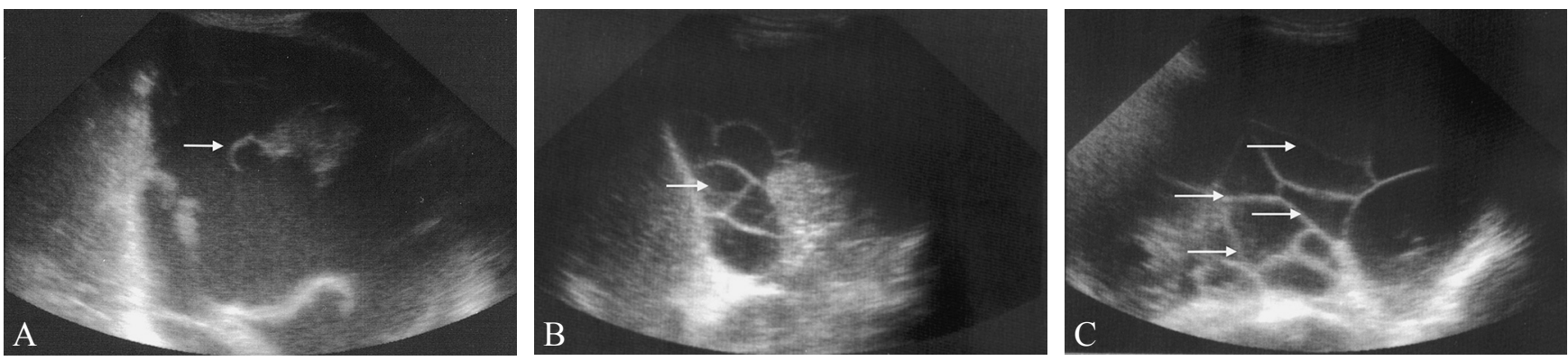

Figure 24.-A-C) Convex probe: fibrin appears as free or organized hyperecogenic lines (arrows) in the pleural effusion.

Ultrasound can determine the presence of pleural effusion but can also help in defining its characteristics and potential causes. We can divide the pleural effusion on the basis of the echogenicity into anechoic, complex nonseptated (particles of variable density interspersed in the fluid), complex septated, or heterogeneously echogenic effusions (fibrin strands or septae organized in a web-like pattern) that may indicate a complicated parapneumonic effusion, or empyema. Exudates which are homogenously hyperechogenic, consisting of diffuse internal echoes with a uniform gray appearance, are usually exudative 32 (Figure 25).
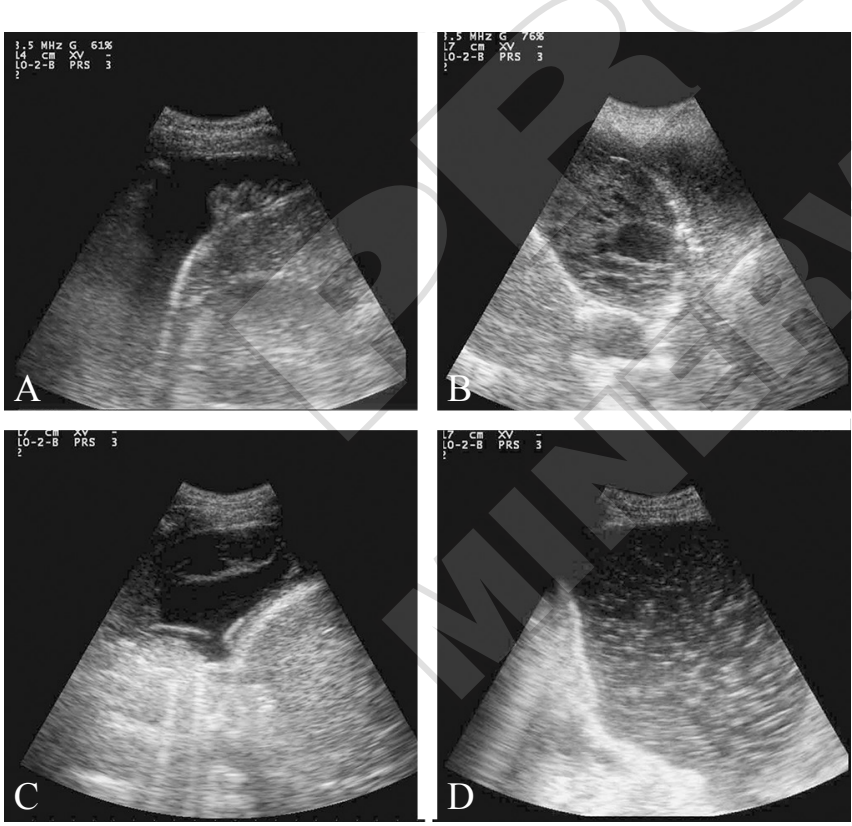

Figure 25.- Convex probe: the pleural effusion on the basis of the echogenicity is divided into: A) anechoic; B) complex non-septated (particles of variable density interspersed in the fluid); C) complex septated (fibrin strands or septae organized in a web-like pattern); and D) homogenously hyperechogenic (consisting of diffuse internal echoes with a uniform gray appearance are usually exudative).

\section{Solid pleural abnormalities}

Pleural ultrasound can detect pleural abnormalities which can be malignant (e.g., a mesothelioma) or benign (e.g., lipomas) and can appear as single or multiple lesions or just as thickening pleura. ${ }^{33}$

Multiple pleural nodules detected in the context of a pleural effusion are highly indicative of pleural cancer.

Lesions between the chest wall and the diaphragm must always be considered potentially tumors, in the presence or even in the absence of an effusion. Diaphragmatic lesions must be considered potentially neoplastic, remembering that they differ from the diaphragmatic pillars which appear as round structures on the diaphragm that modify their shape during respiratory movements, synchronously to diaphragmatic movements (Figure 26, 27).

Ultrasound cannot distinguish the histological nature of the lesions but can only give a probability of benign or malignant (Figure 28, 29).

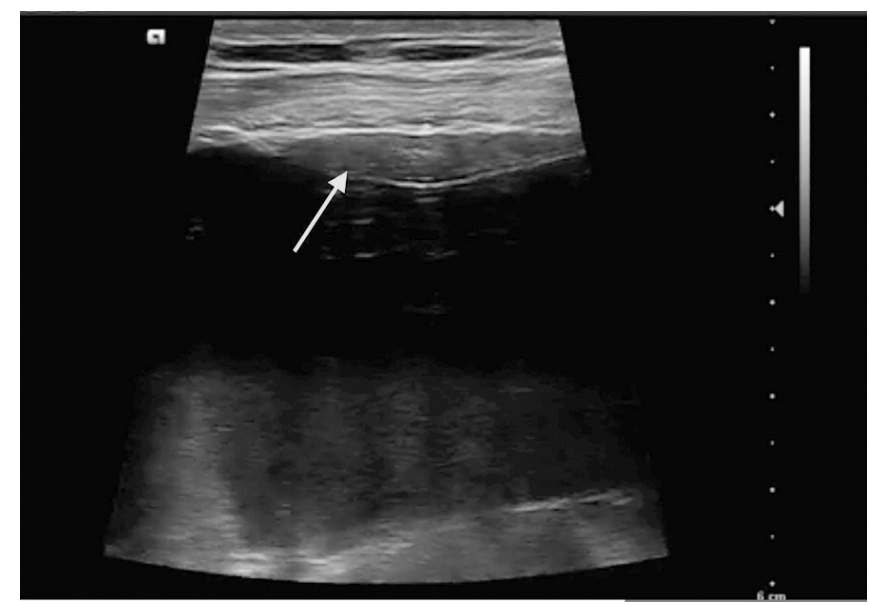

Figure 26.- Linear probe: focal pleural thickening (arrow) with associated pleural effusion. 


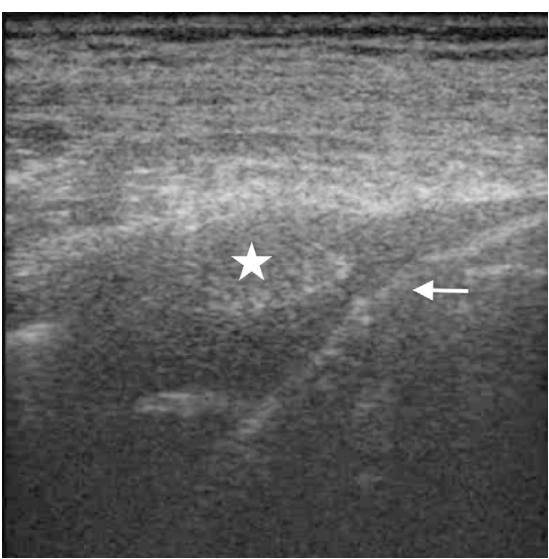

Figure 27.--Linear probe: pleural lesion (star) associated with small pleural effusion that discovers the diaphragm (arrow).

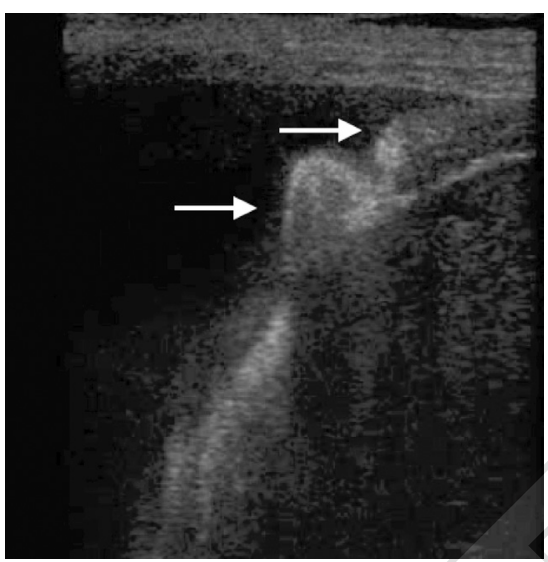

Figure 28.- - Linear probe: diaphragm pilaster (arrows) can be seen as round structures on the pleural surface of diaphragm. During diaphragm moment changes their morphology.

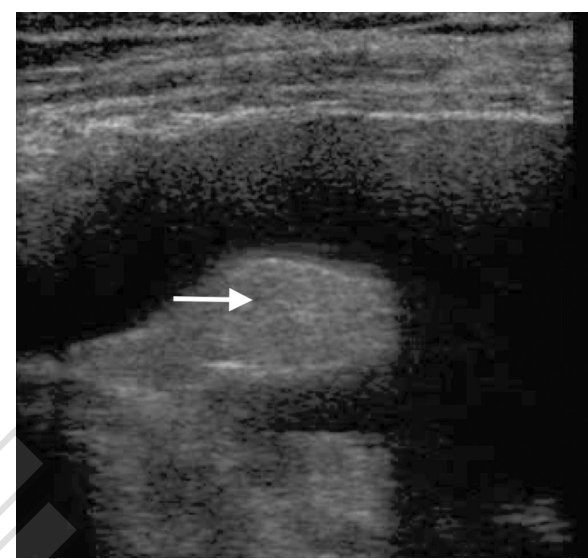

Figure 29.-Linear probe: pleural lipoma (arrow) growth from the surface of diaphragm and pleural effusions.
Pleural thickenings usually appear as focal or disseminated echogenic lesions arising from the visceral or parietal pleura that are greater than $3 \mathrm{~mm}$ in width, with or without irregular margins. There are various echogenicities of thickened pleura. The range of echogenicity varies from hypoechogenic to increased echogenicity and sometimes to highly echogenic shadows indicative of calcification. Pleural thickening can be associated to adhesions. Focal absence of pleural sliding is raising the suspicion of pleural adhesions.

It is important to differentiate a minimal or loculated pleural effusion from a pleural thickening before thoracentesis is performed. Both conditions may have similar US appearances, but there are features useful to distinguish between the two $0^{34}$ (Figure 30A, B).

Pleural lesions are well-defined, hypoechoic or echogenic solid nodular located in the parietal or visceral pleura. These lesions can appear as polypoid pleural nodules
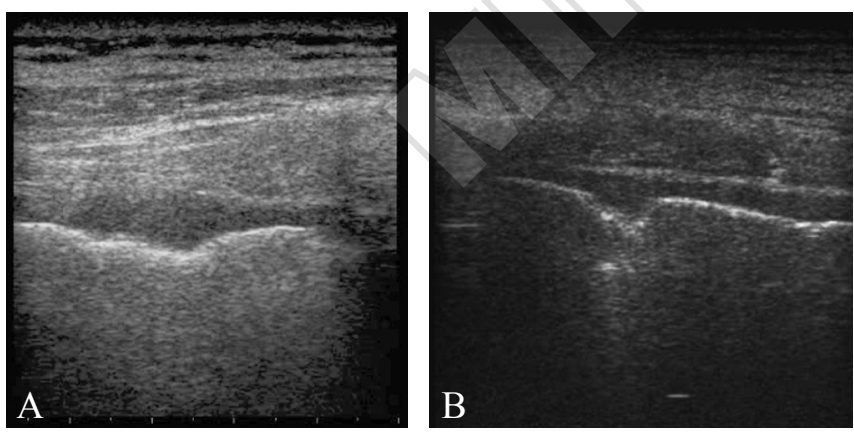

Figure 30.-A, B) Linear probe: diffuse pleural thickening without effusions. or sheet-like pleural thickening combined with pleural effusion (Figure 31A, B). Sometimes, the differentiation between pleural fibrosis and a pleural tumor is difficult even on US.

Parietal lesions appear fixed and sliding can be seen under the lesions, while parenchyma lesions moves together with the lung movement.

Ultrasound is very useful to guide a biopsy needle into the lesions for histopathological diagnosis (Figure 32).

\section{Knowledge of the instruments (knobology and equipment)}

Although US machine size and equipment have changed over time, the basic principles and fundamental functions have remained essentially the same. The fundamental principle of ultrasonography can be traced to approximately 200 years ago when Lazzaro Spallanzani, an Italian biolo-
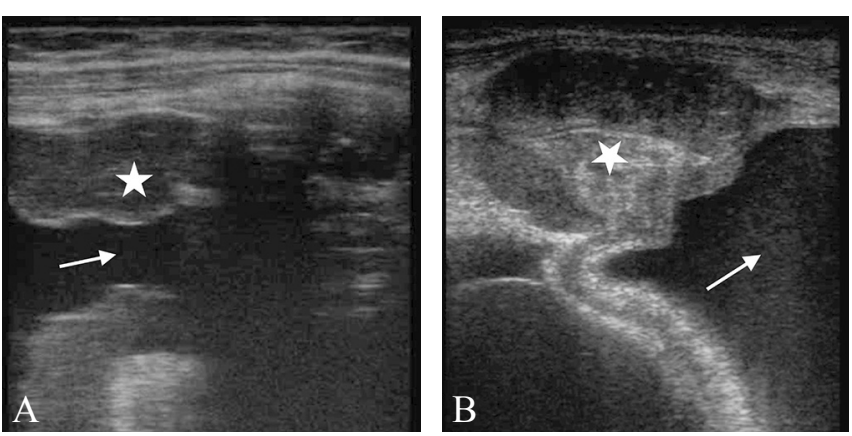

Figure 31.-A, B) Linear probe: pleural lesions (star) and pleural effusions (arrow). 


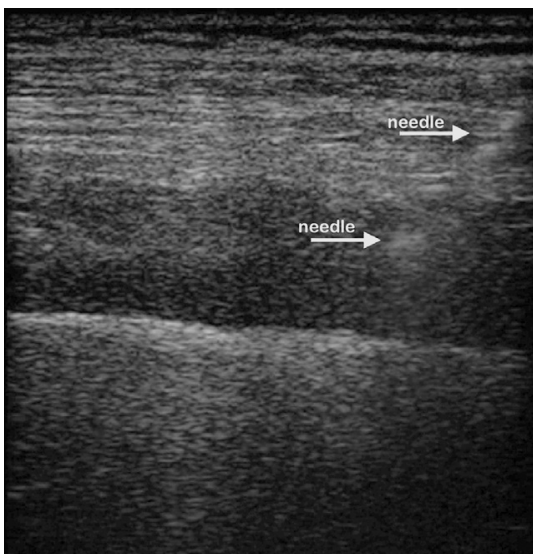

Figure 32.-Linear probe: needle biopsy (arrows) of pleural thickening.

gist, theorized that bats used echolocation to hunt in the dark. ${ }^{35}$ From the 1880 's, when the piezoelectric effect of crystals was first described by Pierre and Jacques Curie, to our days the US machine industry has changed significantly. Today US machines are compact and portable (larger no more than a small laptop computer) and they are used at bedside. Moreover, there are US machines comparable in size to an average cellular phone. Furthermore, there are applications that connect a smart phone to a scanning probe, enabling the operator to perform ultrasonography without an actual US machine. ${ }^{36}$

Medical US is based on the use of high-frequency sound to aid in the diagnosis and treatment of patients. US frequencies range from 2 to approximately $15 \mathrm{MHz}$, although even higher frequencies may be used in some situations.

The US beam originates from the mechanical oscillations of numerous crystals in a transducer, which is excited by electrical pulses (piezoelectric effect). The transducer converts electrical energy into mechanical/sound. Ultrasound transducers work with a range of ultrasound frequencies, termed bandwidth. These frequencies are 2.5$3.5 \mathrm{MHz}$ for general abdominal imaging and 5.0-7.5 MHz for superficial imaging.

US waves are reflected at the surfaces between the tissues of different density proportional to the difference in impedance. If the difference in density is increased, the proportion of reflected sound is increased. If the difference in tissue density is very different, then the sound is completely reflected, resulting in a total acoustic shadow (like with bones, calculi and air).

Echoes are not produced if there is no difference in a tissue density or between tissues. Homogenous fluids like blood, ascites and pleural effusions are seen as echo-free structures.
US functions through the following steps:

- a probe generates a sound pulse that travels through the body.

- waves hit a boundary between tissues (e.g. between fluid and soft tissue, soft tissue and bone);

- some of the waves get reflected back to the probe, while the rest travel on further, until they reach another boundary and get reflected;

- the reflected waves are picked up by the probe and relayed to the machine;

- the machine calculates the distance from the probe to the tissue or to the organ (boundaries) using the speed of sound in the tissue and the time of each echo's return;

- the machine displays the distances and intensities of the echoes on the screen, forming a two-dimensional image.

US machines are made up of different parts with specific functions, such as:

- transducer probe (sends and receives the sound waves);

- central processing unit (CPU) (calculates and contains the electrical power supplies);

- transducer pulse controls (amplitude, frequency and duration of the pulses emitted);

- display for the image obtained by the ultrasound and the CPU;

- keyboard/cursor for inputs data and takes measurements from the display;

- disk storage device to stores the acquired images (optional);

- printer of the image from the displayed data (optional).

Transducer probe

Piezoelectric crystals are located at the footprint of the probe and, except for the sector probe, they are arranged according to the shape of the probe. The footprint is the transmitter and the receiver of the US beam.

Transducer probes have different shapes and sizes. The shape of the probe determines its field of view, and the frequency of emitted sound waves determines how deep the sound waves penetrate and the resolution of the image (Figure 33A, B). High-frequency probes should be used to visualize superficial structures (tendons, muscle, pulmonary pleura, vasculature and so forth), while lowerfrequency probes should be used to visualize deeper structures (abdominal aorta, the gallbladder, the inferior vena cava, pelvic organs and so forth), but this is done at the expense of the resolution.

Transducer probes may contain one or more crystal el- 
ements; in multiple-element probes, each crystal has its own circuit. Multiple-element probes have the advantage that the US beam can be "steered" by changing the timing in which each element gets pulsed; steering the beam is especially important for cardiac US (Figure 34).

Probes are designed to get moved across the surface of the body or be inserted through various openings of the body (vagina, rectum, esophagus) so that they can get closer to the organ being examined for more detailed views. Every US probe have a probe marker (colored light, dot, or a linear ridge that can be easily palpated) along one side of the head of the probe. Markers are important for orientation of the patient's anatomy in relationship to the maker displayed on the US machine screen.

The curvilinear or convex array probe have a frequency range between 2 to $5 \mathrm{MHz}$. It provides a wide, fan-shaped scanning area on the US screen. This type of transducer is mostly used for evaluating deep structures in the abdomen and pelvis. ${ }^{37}$

The linear transducer has a rectangular footprint shape, with a frequency range of 6 to $15 \mathrm{MHz}$. This probe provides detailed anatomical resolution and it is ideal for evaluating superficial structures and to guide needle aspirations.

The intracavitary probe has a curvilinear crystal array with a wide view, but the frequency is much higher (8-13 $\mathrm{MHz}$ ), so the resolution of the images is better than with the curvilinear probe.

The phased or sector array transducer have a frequency range between 1 to $5 \mathrm{MHz}$. The crystal arrangement in the footprint is bundled in the center and fans out creating a pie-like image on the US machine screen. This probe is commonly used for echocardiography.

Some US machines allow the user to change the broadband frequency used during the scan.

\section{Central processing unit}

The central processing unit (CPU) sends electrical currents to the transducer probe to emit sound waves, and also receives the electrical pulses from the probes that were created from the returning echoes. The CPU is involved in processing the data and to obtain the raw data that return as the image on the monitor. The CPU and probes work in synchrony to transmit, receive, and depict sound waves and to produce US images for evaluation.

The US machine receives the beam signal as amplitude, frequency, and the changes of the frequency over time. The two- dimensional gray scale of the image is generated from the amplitude of the echoes. The change of the

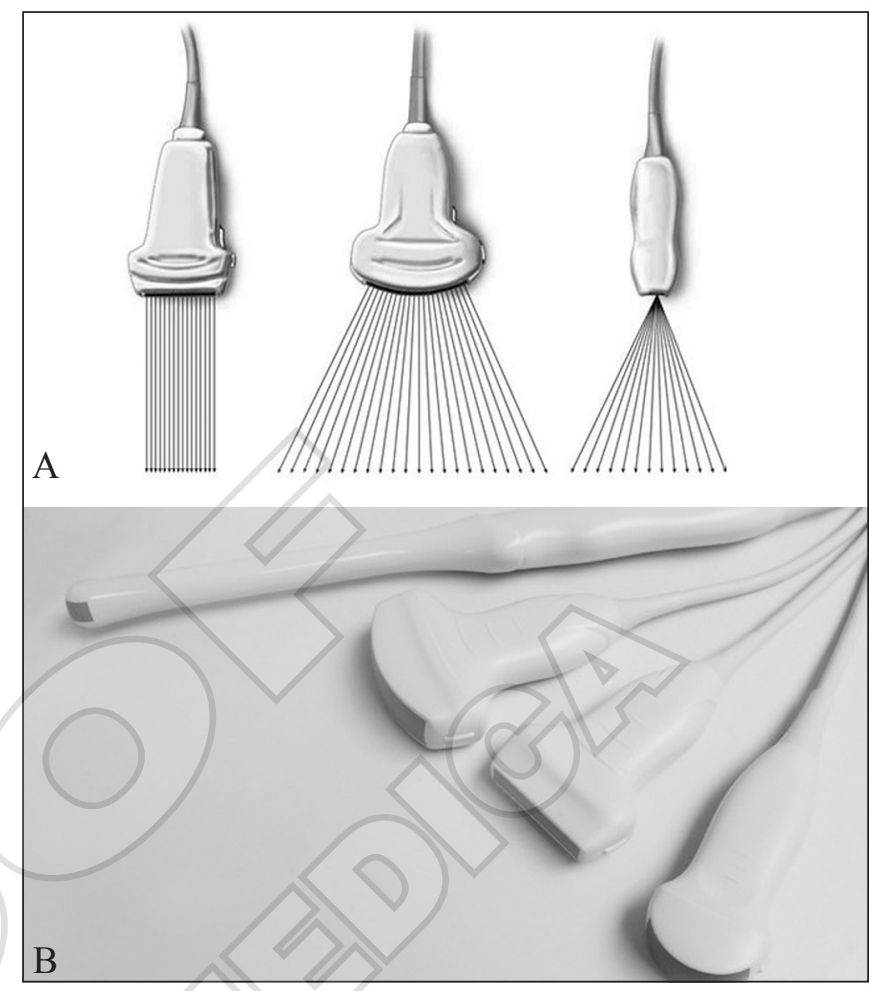

Figure 33.-A, B) Transducer probes have different shapes and sizes. The shape of the probe determines its field of view.

frequency and wavelength of the US echoes from a target in motion is known as the Doppler effect.

Before beginning a scan, it is important to set up the US machine for the type of examination that is being performed. Most modern-day US machines have standard presets for common applications (e.g, abdomen, obstetrics, vascular, musculoskeletal). These presets can be selected via a menu available on the machine and are often programmable based on the user's preferences.

\section{Time gain compensation}

A way to overcome ultrasound attenuation is time gain compensation (TGC), when the signal gain is increased as time passes from the emitted wave pulse. This correction makes equally echogenic tissues look the same, even if they are located at different depths (Figure 33A, B). It is also known that the emitted US wave amplitude gets smaller as it penetrates a tissue, a phenomenon called attenuation. So, one might expect late echoes (from deep layers) to have smaller amplitudes than earlier (superficial) echoes even if those layers have the same echogenicity. If the US image is formed directly by the raw 

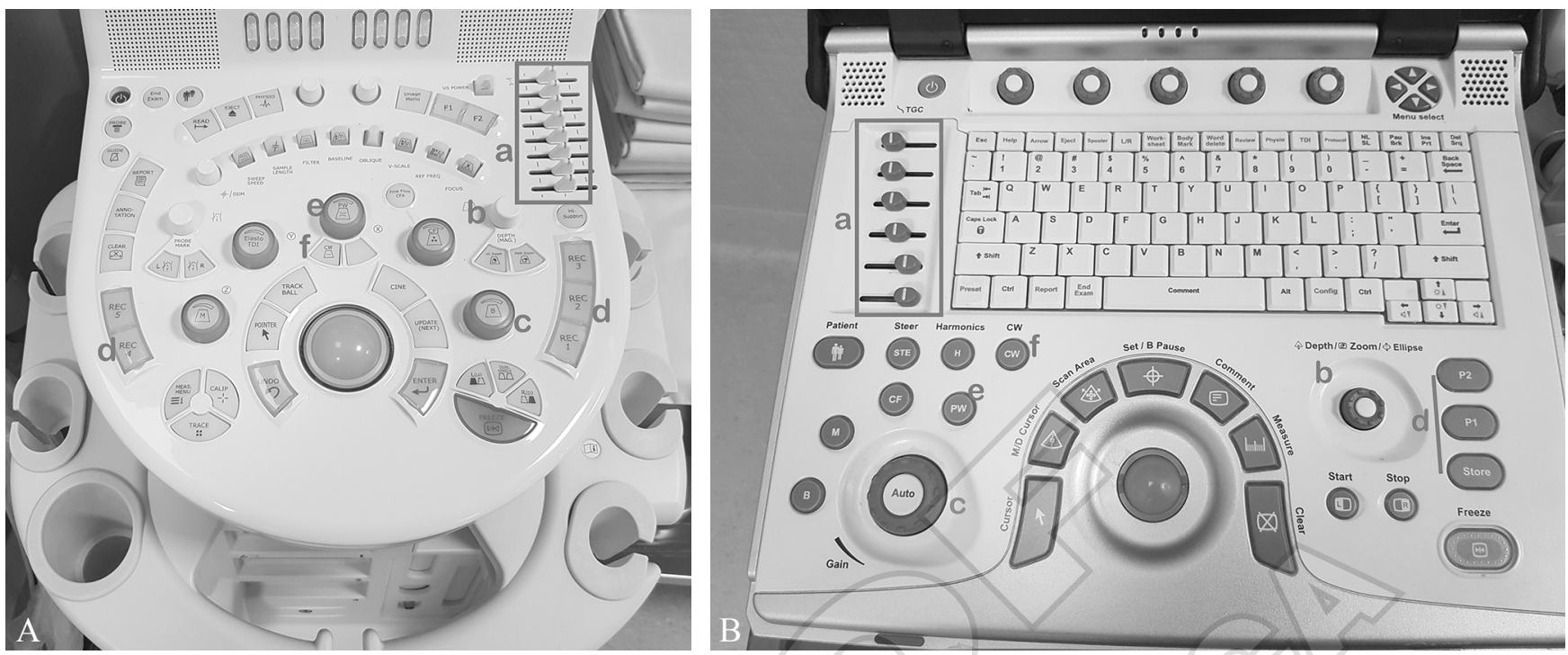

Figure 34.-A, B) Some functions of the US machine: time gain compensation (a), depth (b), gain (c), cine or photo memory (d), pulse Doppler (e), continuous Doppler (f).

returned echoes, image will appear lighter in superficial layers and darker in deep layers, but TGC is used to overcome this artifact. Applying gain to returning echoes from deep structures compensates for this. The operator decides at what level this gain will be applied by the use of the TGC controls.

Transmission power

This function regulates the strength of US beam modifying the amount of energy exciting the crystal. This should be kept to the minimum for required depth penetration to minimize exposure.

Depth

The depth control alters the depth of the field of view. The number of pixels in the image area is fixed so setting the field of view also sets the number of pixels used to represent each square centimeter of the patient (Figure 33A, B). It is recommended to use the smallest depth of field necessary to image the structure of interest. Small depth of field is utilized for superficial structures. Large depth of field is utilized for deeper structures, at FOCUS examinations.

\section{Dynamic range}

The term refers to the range of echoes from strong to weak, available to be displayed on the monitor at a given time (Figure 33A, B). Decreasing dynamic range gives fewer grays and increases contrast. Increasing dynamic range gives a wider range of grays and decreases contrast.

This control is used to magnify structures of interest and is best used in real time or write-zoom mode. Write-zoom affects image quality because the line density and pixels/ unit area are increased. The consequence of these benefits is a decreased field of view.

Cine memory

This represents storage of a number of previous image frames on system freeze. The number of frames retained varies with the manufacturer and with the memory requirement per frame stored. The saved sequence of frames can be reviewed after the examination (Figure 33A, B).

\section{B-mode color}

Many systems allow the echoes (normally displayed in shades of gray) to be displayed in hues of color. This can be useful for an apparent enhancement of contrast resolution, since the human eye can more readily discern small changes in color hue than in shades of gray. The choice of color map appears to be quite subjective; most manufacturers offer a range of different maps for individual preference (Figure 33A, B).

Doppler

Color flow imaging can be used to identify the presence and direction of flow. Pulsed wave Doppler is used to 
provide analysis of the flow at specific sites in the vessel under investigation. When using color flow imaging with pulsed wave Doppler, the color flow/B-mode image is frozen while the pulsed wave Doppler is activated (Figure 33A, B).

Power Doppler is also referred to as energy Doppler, amplitude Doppler and Doppler angiography. The magnitude of the color flow output is displayed rather than the Doppler frequency signal. Power Doppler does not display flow direction or different velocities. It is often used in conjunction with frame averaging to increase sensitivity to low flows and velocities. (Figure 33B). Power Doppler is particularly useful when examining superficial structures, like thyroid, testis, renal grafts and subcutaneous lesions. It may be used to look for tumor vessels, to evaluate tiny low-flow vessels and to detect subtle ischemic areas.

\section{Core basic skills and procedural description (image acquisition)}

Ultrasound examination is traditionally considered a safe and repeatable exam, but its use is highly operator dependent. Because of this, lack of sufficient operator skills could lead to diagnostic errors and to unnecessary tests or interventional procedures. Consequently, according to international recommendations, 2,8 core basic skills and minimum training recommendations for the practice of medical ultrasound and image acquisition are needed to ensure competence of clinicians using ultrasound.

Clinician should have theoretical and practical knowledge on: physics and technology of US, indications and methodology of US examination, normal echographic thoracic anatomy and identification and detection of signs of pleuro-pulmonary pathology.

The use of a systematic approach in few steps is considered essential in achieving such competences and will ensure a high diagnostic accuracy to the US examination.

\section{Performance of an ultrasound examination: procedural steps}

Correct positioning of the patient depending on clinical conditions

The ideal patient position for TUS is critical to obtain valuable diagnostic information and the best image acquisition. Different lung areas and pathological conditions will be better evaluated in decubitus. The sitting or standing position is suitable for assessment of the lateral and poste- rior chest and it is recommended for identification of free fluid pleural effusion or pneumonia. The supine position allows assessment of ventral chest and is recommended to detect pneumothorax.

In both positions, by lifting the patient's arm above the head, or by asking the patient to position his or her hand on the contralateral shoulder, the relative narrow intercostal spaces will be opened, and the subscapular region will be better accessed (this in the sitting position only).

The main factors in determining the correct position for TUS are:

1. the aim of the examination:

- diagnostic TUS: it is a complete ultrasonographic examination in which the clinician evaluates all possible pathological conditions in an organ or a structure, including the possibility to declare "normality." All thoracic surface must be evaluated, both in supine and in sitting position;

- focused TUS: it is an ultrasonographic examination focused to answer specific and clinically relevant diagnostic questions. Patient's position will be chosen according to clinical question, for example supine position to assess presence of pneumothorax, etc.;

- interventional TUS: in this case ultrasonographic examination is ancillary to a diagnostic or therapeutic procedure. In case of thoracentesis, sitting position will be preferred, in case of pleural drainage, supine or sitting position will be chosen, lateral decubitus will be preferred in order to choose best site entry before medical thoracoscopy. ${ }^{38} \mathrm{~Pa}-$ tient should be positioned in the most comfortable position, with adequate support to maintain the requested position for all the procedure time. Clinicians should also evaluate their own comfort during the procedure and ensure proper posture and ergonomics in order to prevent overuse problems; 39

2. the patient's clinical conditions: in a critically ill patient or in ICU, the scanning positions will be limited to supine, semi-recumbent or lateral scans. Posterior thoracic zone could be assessed by lying the patient on his side, or by inserting the transducer between the patient and the mattress.

The clinician determines the thoracic surfaces to be explored and the clinical objectives

When performing TUS, choice of thoracic surface to be explored is related to clinical objective of the examination.

The performance of a diagnostic TUS requires an assessment of both lungs, in order to search for pleuro-parenchymal abnormalities all over the chest's ultrasonographic explorable area, just as for auscultation. Intercostal spaces offer adequate acoustic windows to the underling lung sur- 
face. By moving the probe transversally in the intercostal spaces in a caudocranially way, starting from the upper abdomen to the lung apex, most of the thoracic surfaces will be evaluated, except for dorsal lung segments of upper lobes, acoustically covered by scapula, and mediastinal lung surface, that could be evaluated only endoscopically. However, it has been estimated that more than $70 \%$ of the pleural surface is visible by ultrasound. A comprehensive ultrasonographic assessment of the lungs and adjoining anatomical structures, such as the pleural spaces, parietal pleura, thoracic wall, supra- and infraclavicular regions and the diaphragm, with qualitative and quantitative assessment of abnormality detected, it is obviously timeconsuming and requires patient's cooperation, so it is done with difficultly in an emergency setting.

Focused TUS (FTUS) has the purpose diagnosing or excluding potentially life-threating conditions. In this case, only a limited area of thoracic surface will be quickly evaluated. Many different focused TUS approaches are described in literature, ${ }^{40-42}$ but there is not an international consensus on which performs better. ${ }^{8}$ The protocol described by Laursen et al. ${ }^{41}$ divides each thoracic surface in seven areas (two anteriorly, two laterally, and three posteriorly), each one representing a scanning zones that should be assessed during FTUS:

- zones R1 and L1: the transducer is in the second intercostal space (ICS) a few centimeters lateral to the sternum;

- zone R2: the transducer is in the 4th ICS, a few centimeters lateral to the sternum. The lower border of the pectoralis muscle is the anatomical landmark;

- zone L2: is passage area from lung to heart, reached by moving caudally the transducer from $\mathrm{L} 1$;

- zone R3: is the lateral side border area between upper abdomen and chest cavity. It is identified by placing the transducer on the mid-axillary line, on the lower part of chest. The first step is liver identification, then the transducer is moved cranially to identify the diaphragm and then the lung;

- zone L3: is the lateral side border area between upper abdomen and chest cavity. It is identified by placing the transducer on the posterior axillary line, on the lower part of chest. The first step is to identify the spleen or the left kidney, then the transducer is moved cranially to identify the diaphragm and the lung;

- zones R4 and L4: are identified by placing the transducer in the 3rd ICS, in the midaxillary line;

- zone R5 and L5: is the posterior side border area between upper abdomen and chest cavity. It is identified by placing the transducer on the posterior axillary line, on the lower part of chest. The first step is to identify the spleen or the left kidney, then the transducer is moved cranially to identify the diaphragm and the lung;

- zone R6 and L6: are posterior and are identified by placing the US-probe in the posterior midclavicular line along the medial border of the middle part of the scapulae, while the patient is asked to place his hands on the contralateral shoulders;

- zone R7 and L7: are posterior and are identified by placing the US-probe in the posterior midclavicular line along the medial border of the cranial part of the scapulae, while the patient is asked to place his hands on the contralateral shoulders.

Choice of probe, site of examination, adjustment of machine

During TUS or FTUS, different probes, characterized by different properties, can be used to evaluate the pleura and the lung:

- convex (or curved) array US transducer: wide footprint and frequency of 2-6 MHz. Best pre-set is an abdominal one, without harmonics to enhance artefacts. This permits good tissue penetration, allowing identification of deeper structures such as pleural effusions, atelectatic lung, abdominal organs, and diaphragmatic pleura, but will provide less detail of superficial structures.

- linear array US transducer: wide footprint and frequency of $7-12 \mathrm{MHz}$. Best pre-set is musculoskeletal or vascular ones, without harmonics to enhance artefacts. It can be used to examine more superficial structures in fine detail, including the chest wall, ribs, lymph nodes and the pleural interface. Higher frequency probes are particularly useful in the identification of pneumothorax

- phased array US transducer: small footprint and frequency of 1-5 MHz. Good tissue penetration, it can be used to assess complex pleural effusion.

- microconvex array US transducer: small footprint and frequency 3-9 MHz. It is a universal transducer useful in FTUS or in patients that can be examined only in supine position due to its length. ${ }^{40}$

How to handle the probe:

- clean the probes with an approved antiseptic wipe before and after usage;

- hold the probe with the first three fingers like a pen, while using the other two fingers to get a stable position to patient's body;

- US probe marker will be directed towards the head when acquiring images in a longitudinal plane. Cranial structures will be seen on the left side of the screen closest to the marker; 
- during transversal image acquisition US probe marker will be directed to the right side of the patient. Structures on the patient's right will be seen on the left side of the screen closest to the marker;

- sufficient gel must be placed along the interface between the transducer and the patient's skin;

- the entire transducer surface must be in close contact with patient's body.

Once decided the kind of examination (diagnostic or focused), the objective of the assessment (evaluation of pleural effusion, pneumothorax, lung consolidation, diaphragmatic excursion) and correct probe to dealt with, the exam will start.

Imaging mode will be chosen among the following:

- two-dimensional (2D) or brightness mode (B-mode): most used modality for TUS;

- M-mode: modality used to assess motion of all structure along a single scan line over time. This is chosen by the operator's placement and manipulation of a vertical line through the object of interest. This scanning modality is used for TUS to evaluate the lung for PTX, diaphragmatic motion or inferior vena cava collapsibility;

- Doppler mode: color flow Doppler differentiates objects, usually blood, moving towards (red colour) or away (blue colour) from the transducer. Another Doppler modality called power Doppler is most useful for detection of low-flow states, but its utility is limited to cardiac evaluation in TUS. To utilize color flow or power Doppler mode, operator will start with a B-mode image, then he will place the color doppler box in the area of interest. The size of the color Doppler box should be limited to the area of interest and gain for color Doppler set to an adequate level to minimize noise (it should be set prior to starting examination until only a few, occasional spots of color appear with the transducer off the patient).

\section{Optimization}

The last technical step is image optimization. An inadequate setting of US machine could compromise the image quality and consequently the diagnostic accuracy and therapeutic decisions. Key principles of image acquisition are:

- frequency: according to the probe range of frequencies, it can be modified according to depth of our target (higher frequencies for superficial structure and lower frequencies for deepest structures);

- depth: it should be set to allow complete visualization of the structures analyzed. Usually, the deepest part of the target is positioned at three quarters of the maximum depth of the screen;
- focal point: to optimize the region of interest, it should be placed at the level or just below the desired target for evaluation;

- gain: it allows adjustment of the display brightness of echoes returning to the receiver. When it is correctly adjusted, fluid appears anechoic and solid tissues appear along a spectrum from white to grey, according to tissue properties. An image too white will be over-gained, instead an image too black will be under-gained;

- time gain control: It should be set to ensure a uniform gain across image.

These parameters will vary throughout the US exam to obtain the best image and avoid diagnostic errors.

\section{Systematic approach to patient examination}

We propose a systematic approach to every ultrasound examination, which should include the following:

- evaluate indication and the objectives of the examination;

- evaluate all the other clinical information available, including radiographs, CT scans and previous ultrasound examinations;

- turn on the ultrasound unit;

- enter patient demographics to ensure that images can be saved;

- position the patient according to objective of the examination;

- position the ultrasound screen in order to be comfortably viewed during the examination;

- select probe, preset and adjust depth, focus, gain, TGC to optimize image acquisition. Continuous changes/adaptations of machine/probe settings should be done during the examination, in order to obtain high quality images;

- orientate US probe marker towards the head during longitudinal scans, and to the right of the patient during transversal scans;

- perform a general exploration with longitudinal and transversal scans, in succession, on each hemithorax, along anatomical vertical lines and along intercostal spaces or in specific thoracic zones;

- recognize the normal sonoanatomy of the thorax: in a longitudinal scan, chest wall structures (skin, subcutaneous tissue, muscles) will be placed in the upper part of the image, the superficial surface of the ribs will be represented as two hyperechoic horizontal lines, with posterior shadowing, separated each one by the intercostal muscles. Just below the inferior margin of the cranial rib (the one on the left side of the sonographic image) intercostal neurovascular bundle can be seen. It is of fundamental importance 
for procedures the recognition of intercostal artery with color Doppler. Below the intercostal space and between ribs, a hyperechoic line generates the "bat-sign," namely the pleural line, which represents visceral and parietal pleura. The two pleural layers cannot be distinguished in absence of pathological alterations. Movement of pleural line during respiratory cycle is named "sliding sign." The area below the pleural line is generated by air artefacts and does not represent normal inflated lung;

- recognition of elements of pleural and pulmonary pathology; $3,8,43$

- technical (image acquisition) and cognitive (image interpretation) elements required for competence in pleural ultrasonography:

- identification of fluid: it is an anechoic or hypoechoic area surrounded by thoracic anatomic structures as diaphragm, chest wall, ribs, visceral pleura, normal/ consolidated/atelectatic lung

- localization of fluid: free-flowing effusion are affected by gravity and it varies according to decubitus position. It involves initially costophrenic angle and, as volume increase, it rises up the pleural cavity and gradually compresses lung. Loculated effusion is not affected by gravity and it will be localized according to intercostal spaces in a transverse plane and vertical lines of the thorax in a longitudinal plane;

- quantification of fluid: through formulas for calculation measuring depth of effusion or its longitudinal diameter $^{31}$ or based on the degree of lung atelectasy; ${ }^{44}$

- characterization of pleural effusion according to the 4 sonographic patterns, ${ }^{45}$ often related to transudative or exudative nature of fluid: 46

- anechoic: few particles floating

- complex non-septated: particles of variable density interspersed in the fluid;

- complex septated: fibrin strands or septae organized in a web-like pattern;

- homogeneously hyperechogenic (densely corpuscolated);

- odynamic effects of fluid: evaluation of diaphragmatic motility, degree of lung atelectasis and respiratory lung movement in pleural effusion (sinusoid sign); 19

- ocollateral findings: search for pleural-based nodule or masses or pleural thickening and evaluation of subdiaphragmatic organs (liver, spleen, kidney and ascites), heart, pericardial effusion and great vessels (aorta and inferior vena cava);

- technical and cognitive elements required for competence in lung ultrasonography:3
- knowledge of the basic semiology of lung ultrasound;

- identification and characterization of air artefacts suggestive of the normal aeration pattern: A-lines with sliding sign;

- knowledge of the limitations of not visualizing lung sliding/B lines: subcutaneous emphysema;

- identification and characterization of air artefacts suggestive of alveolar interstitial pattern: number, location and gradient of B lines, presence of subpleural consolidation, white lung;

- identification and characterization of consolidated lung: identification of lung density, presence and characteristics of bronchograms to discriminate among pneumonia (consolidated lung, dynamic air bronchograms, perilesional sonographic interstitial syndrome) or atelectasis (consolidated lung, static and parallel air bronchogram);

- identification and characterization of air artefacts to rule out pneumothorax: presence of sliding sign, B lines or lung pulse;

- identification of echographic signs of pneumothorax:

- use of a linear or microconvex array;

- patient should be in a supine position and the exploration started from the medial (least gravitationallydependent areas) to the lateral side of each hemithorax;

- adjust depth (parietal pleura on the $3 / 4$ of the screen) and focus (on the parietal pleura);

- identification of pathognomonic signs of pneumothorax: presence and localization of lung point(s), absence of lung sliding, B lines and lung pulse, stratosphere sign in M-mode;

- limitation to the identification of the pathognomonic signs: subcutaneous emphysema.

\section{Elaboration of a clinical suspicion}

Thoracic ultrasound can be performed also during or after the physical exam to integrate the information provided by clinical signs and symptoms. Ultrasound, physically based on mechanical waves, is actually an evolved form of percussion, so that the percussive part of physical exam becomes obsolete. Ultrasound can be used to explore the curtain sign and to examine the bilateral excursion of lung bases and to identify any asymmetries. Once suspected, a reduced motility of the hemidiaphragm can be confirmed with an ultrasound study of diaphragm excursion and thickening. ${ }^{47}$ A percussive dullness, in presence of auscultatory silence or bronchial breathing, can be identified as a pleural effusion, an atelectasis or a 
pneumonia, transforming a sign into a diagnosis. Nevertheless, ultrasound can integrate the information provided by lung sounds, but it is not possible to replace the auscultation with ultrasound examination. In facts, dry sounds such as wheezing, groaning and rhonchi, which are an expression of bronchial obstruction, often are associated with ventilated peripheral lung, providing a normal ultrasound pattern.

US can also be useful to identify different causes of crackles: wet crackles can be seen as a congestive interstitial syndrome associated sometimes with basal pleural effusion in congestive heart failure, or with a lung consolidation associated to air bronchograms in pneumonia; dry crackles can be seen as pleural irregularities and thickening given by old pleurisy; Velcro crackles can be seen as an irregular lung surface associated with interstitial syndrome, giving a strong suspicion of an interstitial lung disease.

\section{Indication/planning of interventional procedures}

Thoracic ultrasound is a useful guide for pleuro-parenchimal interventional procedures (see dedicated section), such as thoracentesis, ${ }^{48}$ insertion of chest drainage, ${ }^{45}$ transcutaneous biopsies of the pleura and peripheral lung parenchyma and thoracoscopy. 38,49

\section{Draft clinical report}

According to ESR and EFSUMB joint recommendation, ${ }^{50}$ it is necessary to draft a formal report for all ultrasonographic examinations, as well as to archive both reports and images or videos within hospital information technology (IT) systems.

Availability or reports and media is important in clinical practice as a record of patient status at the time of examination and as a reference for correlative imaging, future comparison and audit.

There is no agreement on the format of the TUS report. A possible way to draft a formal report could be summarized in the following points:

- machine and probes used;

- clinical context (TUS or FTUS);

- type of breathing/oxygen therapy;

- patient's position;

- normal/abnormal patterns.

\section{Theoretical and practical training}

Regardless of the organization that is producing the guidelines, there are certain elements in the TUS education that are common to all documents. These include a short theoretical course, followed on the same occasion, or latter, by a short period of practical activity under supervision, which is then followed by a longer period of independent activity under direct or distant supervision.

For example, EFSUMB describes the syllabus of a course, and assessment form, and then suggest that the trainee should observe at least 25 thoracic ultrasound examinations and perform, under supervision at least 100 examinations on normal patients, 50 examinations on patients with pleural effusions, and 25 thoracocenteses. ${ }^{2}$

Most of the short hands on courses focus on basic theoretical aspects of ultrasound physics, machine settings and image acquisition, with image interpretation based on stored images. Few courses offer the possibility of scanning real patients in real clinical circumstances. The content of the hands-on courses is thus largely variable and also depends on local availability of scanners, simulators, trained trainers and other facilities. The teaching methods involved at these courses are usually classic, classroom, didactic lectures, with few courses offering web-based learning. ${ }^{51}$ Despite clear a preference of today's doctors for a learner-centered education method, there is only just one study which measured the effect of two educational methods in TUS. ${ }^{52}$ Furthermore, there is no agreement on a standard course content or curriculum, nor a standard process of evaluation, and many courses are not offering any follow up mentoring program or re-training.

\section{Training on simulators}

Simulation emerged as extremely useful in TUS education, because it provides excellent opportunities for standardized teaching and assessment, without creating discomfort for patients or volunteers, or putting them at risk. ${ }^{53}$ Learners can practice again and again certain image acquisition maneuvers and can see some pathological images, which they may not otherwise scan during their training on volunteers, or on selected patients.

There are available a number of commercial thoracic ultrasound simulators, including online simulators, mannequins and phantoms. All have various capabilities and, in general, are expensive. A mannequin-based simulator can display both the anatomical structure (in an augmented reality window) and the ultrasound image from a stored dataset, and can help the student to learn complex image acquisition techniques. Moreover, these complex simulators can give some form of feedback both on the correctness of the image acquisition technique and the image interpretation and can also run clinical scenarios. 
The phantom type simulators offer a real ultrasound image of a real structure that have similarities with thoracic structures. They are particularly useful for practicing ultrasound guided procedures. There are expensive commercially available phantoms, but there are also good descriptions of "do it yourself" (DIY) cheap phantoms, which should not be ignored.

A DIY ultrasound phantom compendium which extends to all body ultrasound application is available from www. ultrasoundtraining.com.au/foamus/diy-phantom-compendium. 54,55

Most of the simulators are used only during hands on, small group, formal courses. In the aviation industry, for example, the best use of simulators is not for small group courses, but for one-to-one training, when the student and the trainer can spend ample time together and repeat again and again and reflect on certain more difficult maneuvers. The principle of a "one-to-one" training on a simulator is not widely applied in TUS, probably because there is a mismatch between demand and supply in terms of access to simulators. Although a lot of training should be done on a simulator, in Europe simulators are available only in few centers with "closed" access, i.e. access only available as part of a course organized by that institution. In between courses most of these simulators are idle. Opening up access to underused simulators through the creation of a simulator network with a booking app similar to Airbnb and booking times (and prices) available to anyone who wants to rent, and practice may improve the access to expensive simulators and may bring some return of the money invested in their purchase.

Mentorship programs

Full competency in TUS cannot be achieved only at a short hands-on course or in few days, no matter how well a particular course is organized, and how good and skilled the teachers are. These courses should be only the starting point of additional supervised practice, until full competency is achieved. The problem of mentorship is usually left to be sorted by the learner, and this component is usually deficient in the learning process. For example, a national survey in the UK showed that $59 \%$ (107/171) of all respondents are 'never' or 'rarely' supervised when performing a TUS after being certified as level 1 competent. 56

We strongly believe that the courses should have attached a structured component of additional supervision, otherwise they are only a "presentation" course of TUS technique, rather than a "training" TUS course. Answering this need requires additional effort and investment from the course organizers. Educational bodies should recognize the necessity for this investment and should commit to it, before bringing in strict rules for course accreditation. There is, however a clear need of having an accreditation system for the courses available, so that the learners know exactly what they are signing and paying for.

\section{Quantitative and qualitative assessment}

There is also a lack of a uniform exam or certification process for TUS. There is agreement that completing a course and a number of procedures does not equate with competency in TUS, but there is little evidence on what can be offered as a certification process or test.

Achieving competency only on the basis of a number of examinations performed is criticized for many reasons. Although the learning curve for TUS is steep, it is known that from a self-selected cohort of learners some will achieve mastery sooner than others, and very few will never reach the plateau of the curve. Ideally all learners should be certified with a similar level of expertise, not for a similar number of examinations. It is useful to propose quantitative assessment. In the consensus document it was:

- 100 clinical examinations with chest ultrasound;

- 10 echo-guided thoracentesis;

- 5 echo-guided insertions of chest tube.

Competency in TUS requires knowledge in at least three distinct domains, which are scanner capabilities, image acquisition and image interpretation. These can be tested individually or, better, as a whole. In clinical practice it is also important the understanding of correlations between different patterns and other clinical findings, plus the limitations of the examination in certain clinical contexts. There is therefore a need for different "clinical areas" of certification. Only recently, in the UK the pleural physicians decided that for both ultrasound and pleural procedural skills, to divide competency into four broad categories: emergency operator, basic operator, intermediate operator and advanced operator. ${ }^{57}$

A useful assessment tool should provide a relevant, valid, and feasible method for evaluation of competence in TUS across multiple specialties, however not many are available. Lung ultrasound-objective and structured assessment of ultrasound (LUS-OSAUS), for example, has been recently proposed as an assessment tool that can differentiate between levels of competence, and which allows for a standardized, but more individualized assessment of competence than few other tools available. ${ }^{58-61}$ Probably 
in the future technology will help the certification process UGSTAT (the Ultrasound-Guided Thoracentesis Skills and Tasks Assessment Test: an 11-domain, 100-point assessmentsheet in line with British Thoracic Society Guidelines) with apps and games based on TUS scenarios, similar to other developments. ${ }^{62}$

\section{Conclusions}

Training in TUS remains varied in different regions and countries. There is a need for a clear, simple pathway for recognition of competence in TUS and further research work is necessary in this area. The evidence so far, together with expert opinions would indicate that good TUS training should involve a short, intense, student orientated course with a standardized theoretical and a practical component, preferable on a high-end simulator, followed by a structured program of mentorship, locally or from a distance. ${ }^{63}$ Competency is not about the number of procedures, but more about doing the procedure correctly every time, and interpreting the results correctly in the clinical context, while knowing the limitations of TUS and the moment when to ask for expert advice. A standard competency pathway would require intense collaborative work among academic societies and a supporting infrastructure for accredited courses, access to simulators and mentorship supervision. Further collaborative work within dedicated training networks may help achieve this goal.

1. Havelock T, Teoh R, Laws D, Gleeson F; BTS Pleural Disease Guideline Group. Pleural procedures and thoracic ultrasound: British Thoracic Society Pleural Disease Guideline 2010. Thorax 2010;65(Suppl 2):ii61-76.

2. EFSUMB - European Federation of Societies for Ultrasound in Medicine and Biology. "Building a European Ultrasound Community" Minimum Training Requirements for the Practice of Medical Ultrasound in Europe. Appendix 11: Thoracic Ultrasound; 2009 [Internet]. Available from: www.efsumb.org/guidelines/2009-04-14apx11.pdf [cited 2018, Nov 12].

3. Mayo PH, Beaulieu Y, Doelken P, Feller-Kopman D, Harrod C, Kaplan A, et al. American College of Chest Physicians/La Société de Réanimation de Langue Française statement on competence in critical care ultrasonography. Chest 2009;135:1050-60.

4. Ruddox V, Norum IB, Stokke TM, Edvardsen T, Otterstad JE. Focused cardiac ultrasound by unselected residents-the challenges. BMC Med Imaging 2017; 17:22.

5. Galarza L, Wong A, Malbrain ML. The state of critical care ultrasound training in Europe: A survey of trainers and a comparison of available accreditation programmes. Anaesthesiol Intensive Ther 2017;49:382-6.

6. Williamson JP, Twaddell SH, Lee YC, Salamonsen M, Hew M, Fielding $\mathrm{D}$, et al. Thoracic ultrasound recognition of competence: A position paper of the Thoracic Society of Australia and New Zealand. Respirology 2017;22:405-8.
7. Laursen CB, Rahman NM, Volpicelli G, editors. Thoracic Ultrasound. Sheffield, UK: European Respiratory Society; 2018.

8. Volpicelli G, Elbarbary M, Blaivas M, Lichtenstein DA, Mathis G, Kirkpatrick AW, et al.; International Liaison Committee on Lung Ultrasound (ILC-LUS) for International Consensus Conference on Lung Ultrasound (ICC-LUS). International evidence-based recommendations for point-of-care lung ultrasound. Intensive Care Med 2012;38:577-91.

9. Pietersen PI, Madsen KR, Graumann O, Konge L, Nielsen BU, Laursen CB. Lung ultrasound training: a systematic review of published literature in clinical lung ultrasound training. Crit Ultrasound J 2018;10:23.

10. Mozzini C, Fratta Pasini AM, Garbin U, Cominacini L. Lung ultrasound in internal medicine: training and clinical practice. Crit Ultrasound J 2016;8:10.

11. Lichtenstein DA. BLUE-protocol and FALLS-protocol: two applications of lung ultrasound in the critically ill. Chest 2015;147:1659-70.

12. Lichtenstein $D$, Mezière G, Seitz J. The dynamic air bronchogram. A lung ultrasound sign of alveolar consolidation ruling out atelectasis. Chest 2009;135:1421-5.

13. Volpicelli G. Lung sonography. J Ultrasound Med 2013;32:165-71.

14. Filopei J, Siedenburg H, Rattner P, Fukaya E, Kory P. Impact of pocket ultrasound use by internal medicine housestaff in the diagnosis of dyspnea. J Hosp Med 2014;9:594-7.

15. Wongwaisayawan S, Suwannanon R, Sawatmongkorngul S, Kaewlai R. Emergency Thoracic US: the Essentials. Radiographics 2016;36:640-59.

16. Lichtenstein DA, Menu Y. A bedside ultrasound sign ruling out pneumothorax in the critically ill. Lung sliding. Chest 1995;108:1345-8.

17. Lichtenstein DA, Lascols N, Prin S, Mezière G. The "lung pulse": an early ultrasound sign of complete atelectasis. Intensive Care Med 2003;29:2187-92.

18. Lichtenstein D, Mezière G, Biderman $P$, Gepner $A$. The "lung point": an ultrasound sign specific to pneumothorax. Intensive Care Med 2000;26:1434-40.

19. Lichtenstein D. Lung ultrasound in the critically ill. Curr Opin Crit Care 2014;20:315-22.

20. Francisco MJ, Rahal A, Vieira FA, Silva PS, Funari MB. Advances in lung ultrasound. Einstein (Sao Paulo) 2016;14:443-8.

21. Dietrich CF, Mathis G, Blaivas M, Volpicelli G, Seibel A, Wastl D, et al. Lung B-line artefacts and their use. J Thorac Dis 2016;8:1356-65.

22. Lichtenstein DA, Lascols N, Mezière G, Gepner A. Ultrasound diagnosis of alveolar consolidation in the critically ill. Intensive Care Med 2004;30:276-81.

23. Qureshi NR, Rahman NM, Gleeson FV. Thoracic ultrasound in the diagnosis of malignant pleural effusion. Thorax 2009;64:139-43.

24. Ho MC, Ker CR, Hsu JH, Wu JR, Dai ZK, Chen IC. Usefulness of lung ultrasound in the diagnosis of community-acquired pneumonia in children. Pediatr Neonatol 2015;56:40-5.

25. Slater A, Goodwin M, Anderson KE, Gleeson FV. COPD can mimic the appearance of pneumothorax on thoracic ultrasound. Chest 2006; 129:545-50.

26. Rose G, Siadecki S, Tansek R, Baranchuk N, Saul T. A novel method of assessing for lung sliding using Doppler imaging. Am J Emerg Med 2017;35:1738-42.

27. Porcel JM, Light RW. Diagnostic approach to pleural effusion in adults. Am Fam Physician 2006;73:1211-20.

28. Mayo PH, Doelken P. Pleural ultrasonography. Clin Chest Med 2006;27:215-27.

29. Han J, Xiang H, Ridley WE, Ridley LJ. Jellyfish sign: pleural effusion. J Med Imaging Radiat Oncol 2018;62(Suppl 1):33.

30. Lee FC. The Curtain Sign in Lung Ultrasound. J Med Ultrasound 2017;25:101-4

31. Vignon $\mathrm{P}$, Chastagner C, Berkane V, Chardac E, François B, Normand 
$\mathrm{S}$, et al. Quantitative assessment of pleural effusion in critically ill patients by means of ultrasonography. Crit Care Med 2005;33:1757-63.

32. Saguil A, Wyrick K, Hallgren J. Diagnostic approach to pleural effusion. Am Fam Physician 2014;90:99-104.

33. Porcel JM. Pleural ultrasound for clinicians. Rev Clin Esp 2016;216:427-35. Spanish.

34. Soldati G, Copetti R. Ecografia toracica - Thoracic ultrasound. Edizioni Medico Scientifiche; 2015.

35. Hoffmann B, Rumsey H, Nixon MS. Physics and Technical Facts for the Beginner; 2008 [Internet]. Available from: www.acep.org/sonoguide/ physics.html [cited 2018, Nov 12].

36. Kendall JL, Hoffenberg SR, Smith RS. History of emergency and critical care ultrasound: the evolution of a new imaging paradigm. Crit Care Med 2007;35(Suppl):S126-30.

37. Bahner D, Blaivas M, Cohen HL, Fox JC, Hoffenberg S, Kendall J, et al.; American Institute of Ultrasound in Medicine. AIUM practice guideline for the performance of the focused assessment with sonography for trauma (FAST) examination. J Ultrasound Med 2008;27:313-8.

38. Marchetti G, Valsecchi A, Indellicati D, Arondi S, Trigiani M, Pinelli $\mathrm{V}$. Ultrasound-guided medical thoracoscopy in the absence of pleural effusion. Chest 2015;147:1008-12.

39. Wareluk P, Jakubowski W. Evaluation of musculoskeletal symptoms among physicians performing ultrasound. J Ultrason 2017;17:154-9.

40. Lichtenstein DA, Mezière GA. Relevance of lung ultrasound in the diagnosis of acute respiratory failure: the BLUE protocol. Chest 2008; 134:117-25.

41. Laursen CB, Sloth E, Lambrechtsen J, Lassen AT, Madsen PH, Henriksen DP, et al. Focused sonography of the heart, lungs, and deep veins identifies missed life-threatening conditions in admitted patients with acute respiratory symptoms. Chest 2013;144:1868-75.

42. Laursen CB, Sloth E, Lassen AT, Christensen R, Lambrechtsen J, Madsen $\mathrm{PH}$, et al. Point-of-care ultrasonography in patients admitted with respiratory symptoms: a single-blind, randomised controlled trial. Lancet Respir Med 2014;2:638-46.

43. Zanforlin A, Giannuzzi R, Nardini S, Testa A, Soldati G, Copetti R, et al. The role of chest ultrasonography in the management of respiratory diseases: document I. Multidiscip Respir Med 2013;8:54.

44. Smargiassi A, Inchingolo R, Zanforlin A, Valente S, Soldati G, Corbo GM. Description of free-flowing pleural effusions in medical reports after echographic assessment. Respiration 2013;85:439-41.

45. Sperandeo M, Filabozzi P, Varriale A, Carnevale V, Piattelli ML, Sperandeo $\mathrm{G}$, et al. Role of thoracic ultrasound in the assessment of pleura and pulmonary diseases. J Ultrasound 2008;11:39-46.

46. Yang PC, Luh KT, Chang DB, Wu HD, Yu CJ, Kuo SH. Value of sonography in determining the nature of pleural effusion: analysis of 320 cases. AJR Am J Roentgenol 1992;159:29-33.

47. Sferrazza Papa GF, Pellegrino GM, Di Marco F, Imeri G, Brochard L, Goligher E, et al. A Review of the Ultrasound Assessment of Diaphragmatic Function in Clinical Practice. Respiration 2016;91:403-11.

48. Dancel R, Schnobrich D, Puri N, Franco-Sadud R, Cho J, Grikis L, et al.; Society of Hospital Medicine Point of Care Ultrasound Task Force. Recommendations on the Use of Ultrasound Guidance for Adult Thoracentesis: A Position Statement of the Society of Hospital Medicine. J Hosp Med 2018;13:126-35.
49. Marchetti G, Arondi S, Baglivo F, Lonni S, Quadri F, Valsecchi A, et al. New insights in the use of pleural ultrasonography for diagnosis and treatment of pleural disease. Clin Respir J 2018;12:1993-2005.

50. European Society of Radiology (ESR); European Federation of Societies for Ultrasound in Medicine and Biology (EFSUMB). Joint ESREFSUMB recommendation on archiving and reporting ultrasound examinations. Insights Imaging 2013;4:525-6.

51. Gargani L, Sicari R, Raciti M, Serasini L, Passera M, Torino C, et al. Efficacy of a remote web-based lung ultrasound training for nephrologists and cardiologists: a LUST trial sub-project. Nephrol Dial Transplant 2016;31:1982-8. [Internet]

52. Edrich T, Stopfkuchen-Evans M, Scheiermann P, Heim M, Chan W, Stone MB, et al. A Comparison of Web-Based with Traditional Classroom-Based Training of Lung Ultrasound for the Exclusion of Pneumothorax. Anesth Analg 2016;123:123-8.

53. McSparron JI, Michaud GC, Gordan PL, Channick CL, Wahidi MM, Yarmus LB, et al.; Skills-based Working Group of the American Thoracic Society Education Committee. Simulation for Skills-based Education in Pulmonary and Critical Care Medicine. Ann Am Thorac Soc 2015;12:579-86.

54. Gómez Betancourt M, Moreno-Montoya J, Barragán González AM, Ovalle JC, Bustos Martínez YF. Learning process and improvement of point-of-care ultrasound technique for subxiphoid visualization of the inferior vena cava. Crit Ultrasound J 2016;8:4.

55. Wilson J, Myers C, Lewiss RE, Wilson J. A low-cost, easy to make ultrasound phantom for training healthcare providers in pleural fluid identification and task simulation in ultrasound-guided thoracentesis. Vis J Emerg Med 2017;8:80-1.

56. Sivakumar P, Kamalanathan M, Collett AS, Ahmed L. Thoracic ultrasound experiences among respiratory specialty trainees in the UK. Clin Med (Lond) 2017;17:408-11.

57. Evison M, Blyth KG, Bhatnagar R, Corcoran J, Saba T, Duncan T, et al. Providing safe and effective pleural medicine services in the UK: an aspirational statement from UK pleural physicians. BMJ Open Respir Res 2018;5:e000307.

58. Skaarup SH, Laursen CB, Bjerrum AS, Hilberg O. Objective and Structured Assessment of Lung Ultrasound Competence. A Multispecialty Delphi Consensus and Construct Validity Study. Ann Am Thorac Soc 2017; 14:555-60.

59. Salamonsen M, McGrath D, Steiler G, Ware R, Colt H, Fielding D. A new instrument to assess physician skill at thoracic ultrasound, including pleural effusion markup. Chest 2013;144:930-4.

60. Patrawalla P, Eisen LA, Shiloh A, Shah BJ, Savenkov O, Wise W, et al. Development and Validation of an Assessment Tool for Competency in Critical Care Ultrasound. J Grad Med Educ 2015;7:567-73.

61. Todsen T, Tolsgaard MG, Olsen BH, Henriksen BM, Hillingsø JG, Konge L, et al. Reliable and valid assessment of point-of-care ultrasonography. Ann Surg 2015;261:309-15.

62. Monroe KS, Evans MA, Mukkamala SG, Williamson JL, Jabaley CS, Mariano ER, et al. Moving anesthesiology educational resources to the point of care: experience with a pediatric anesthesia mobile app. Korean J Anesthesiol 2018;71:192-200.

63. Corbetta L, Patelli M. Executive Summary of Training and Competence Standards for the Interventional Pulmonology Master Program in Italy. J Bronchology Interv Pulmonol 2018;25:6-8.

Conflicts of interest.-The authors certify that there is no conflict of interest with any financial organization regarding the material discussed in the manuscript. Article first published online: November 27, 2018. - Manuscript accepted: November 7, 2018. - Manuscript received: November 6, 2018. 